\title{
P2X7R-mediated autophagic impairment contributes to central sensitization in a chronic migraine model with recurrent nitroglycerin stimulation in mice
}

Li Jiang ${ }^{1}$, Yixin Zhang ${ }^{1}$, Feng Jing ${ }^{2}$, Ting Long ${ }^{3}$, Guangcheng Qin ${ }^{4}$, Dunke Zhang ${ }^{4}$, Lixue Chen ${ }^{4}$ and Jiying Zhou ${ }^{1 *}$ (D)

\begin{abstract}
Background: Central sensitization is an important pathophysiological mechanism of chronic migraine (CM). According to our previous studies, microglial activation and subsequent inflammation in the trigeminal nucleus caudalis (TNC) contribute to the central sensitization. The P2X7 receptor (P2X7R) is a purinergic receptor expressed in microglia and participates in central sensitization in chronic pain, but its role in CM is unclear. Numerous studies have shown that P2X7R regulates the level of autophagy and that autophagy affects the microglial activation and inflammation. Recently, autophagy has been shown to be involved in neuropathic pain, but there is no information about autophagy in CM. Therefore, the current study investigated the role of P2X7R in CM and its underlying mechanism, focusing on autophagy regulation.

Methods: The CM model was established by repeated intraperitoneal injection of nitroglycerin (NTG) in mice. A Von Frey filament and radiant heat were used to assess the mechanical and thermal hypersensitivity. Western blotting and immunofluorescence assays were performed to detect the expression of P2X7R, autophagy-related proteins, and the cellular localization of P2X7R. To determine the role of P2X7R and autophagy in CM, we detected the effects of the autophagy inducer, rapamycin (RAPA) and P2X7R antagonist, Brilliant Blue G (BBG), on pain behavior and the expression of calcitonin gene-related peptide (CGRP) and c-fos. In addition, the effect of RAPA and BBG on microglial activation and subsequent inflammation were investigated.

Results: The expression of P2X7R was increased and was mainly colocalized with microglia in the TNC following recurrent NTG administration. The autophagic flux was blocked in CM, which was characterized by upregulated LC3-II, and accumulated autophagy substrate protein, p62. RAPA significantly improved the basal rather than acute hyperalgesia. BBG alleviated both basal and acute hyperalgesia. BBG activated the level of autophagic flux. RAPA and BBG inhibited the activation of microglia, limited the inflammatory response, and reduced the expression of CGRP and c-fos.
\end{abstract}

(Continued on next page)

\footnotetext{
* Correspondence: zheadache@163.com

'Department of Neurology, The First Affiliated Hospital of Chongqing

Medical University, 1st You Yi Road, Yuzhong District, Chongqing 400016,

China

Full list of author information is available at the end of the article
}

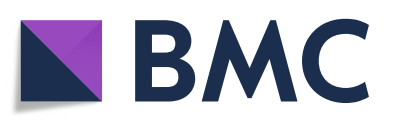

(- The Author(s). 2021 Open Access This article is licensed under a Creative Commons Attribution 4.0 International License, which permits use, sharing, adaptation, distribution and reproduction in any medium or format, as long as you give appropriate credit to the original author(s) and the source, provide a link to the Creative Commons licence, and indicate if changes were made. The images or other third party material in this article are included in the article's Creative Commons licence, unless indicated otherwise in a credit line to the material. If material is not included in the article's Creative Commons licence and your intended use is not permitted by statutory regulation or exceeds the permitted use, you will need to obtain permission directly from the copyright holder. To view a copy of this licence, visit http://creativecommons.org/licenses/by/4.0/. The Creative Commons Public Domain Dedication waiver (http://creativecommons.org/publicdomain/zero/1.0/) applies to the data made available in this article, unless otherwise stated in a credit line to the data. 
(Continued from previous page)

Conclusions: Our results demonstrate the dysfunction of the autophagic process in CM. Activated autophagy may have a preventive effect on migraine chronification. P2X7R contributes to central sensitization through mediating autophagy regulation and might become a potential target for CM.

Keywords: P2X7R, Autophagy, Microglia, Inflammation, Central sensitization, Chronic migraine

\section{Background}

Chronic migraine $(\mathrm{CM})$ is the most common disorder of chronic daily headache, which is manifested as headache for at least 15 days a month [1]. Due to the frequent and severe headache attacks, CM has a high disability rate and great disease burden. At present, the management of $\mathrm{CM}$ is still a major challenge for neurologists due to the limited treatment choices, inadequate evidence of prophylactics, and poor treatment response [2]. Improved knowledge of the precise pathogenesis might benefit the development of new therapeutic targets for CM.

It is widely accepted that central sensitization, which is characterized by increased excitability of neurons in the central trigeminal pathway, plays a critical role in the development and progression of CM [3, 4]. Previous research associated with the mechanism underlying central sensitization has mainly focused on neurons, while recent studies confirm that microglia in the central nervous system (CNS) are also involved in this process $[5,6]$. Our previous works have shown that the activated microglia in the trigeminal nucleus caudalis (TNC) area contribute to central sensitization in the CM by releasing inflammatory mediators and neurotrophic factors and subsequent microglia-neuron interactions [7-10].

The theory that purinergic signaling participates in the pathophysiology of migraine was proposed by Geoffrey Burnstock in the 1980 [11]. The purinergic receptors, P2X4R and P2Y12R, were confirmed to contribute to the pathogenesis of $\mathrm{CM}$ according to our previous works $[7,9]$. P2X7R, an ionotropic purinergic receptor that is widely expressed in the microgli a[12], has been reported to be involved in the cancer pain, neuropathic pain, and inflammatory pain by mediating the microglial activation and the inflammatory response [13-15]. In the acute migraine model established by single nitroglycerin (NTG) injection, P2X7R inhibition attenuated NTG-induced thermal hyperalgesia and $\mathrm{c}$-fos expression in the TNC area, suggesting that P2X7R might participate in the pathophysiology of migraine [16]. However, the role and specific mechanism of P2X7R in the chronic process of $\mathrm{CM}$ have not been explored to date.

Autophagy is an ubiquitous and conserved cellular process in which damaged organelles and abnormal proteins are delivered to lysosomes for degradation [17]. This process is essential to maintain the homeostasis of the cellular environment and achieve the requirements of cellular metabolism as well as organelle turnover [18]. Autophagy is regulated by immune signals [19]. Numerous studies have indicated the critical role of P2X7R in autophagy regulation [20-23]. The P2X7R-mediated autophagy modulation has been confirmed to participate in the pathophysiological process of amyotrophic lateral sclerosis (ALS) [22], Duchenne muscular dystrophy [23], and status epilepticus [21]. Meanwhile, extensive evidence has revealed the interaction between autophagy and inflammatory processes. Autophagy can regulate the activation of microglia and subsequent inflammatory response [24, 25]. Dysfunctional autophagy promotes microglial activation through regulating the production of IL1 $\beta$ and IL18 via NLRP3 degradation, and it contributes to neuroinflammation in Alzheimer's disease (AD) [26]. We have previously confirmed the pivotal role of NLRP3 inflammasome activation in the central sensitization in CM [10]. In addition, altered expression of the autophagy marker in neuropathic pain (Nep) indicates that autophagy is involved in the modulation of pain processing $[27,28]$. However, the effect of autophagy on migraine has not been identified. The relationship between autophagy and P2X7R in $\mathrm{CM}$ has not been previously investigated.

Therefore, in this study, we evaluated the level of autophagy in CM and identified its effect on pain relief. Then, we investigated the role of P2X7R in CM and explored the underlying molecular mechanisms, mainly focusing on autophagy regulation. Our data revealed the blockage of autophagy in NTG-induced CM and the protective effect of autophagy on CM. Microglial P2X7R was upregulated following NTG injection and contributed to central sensitization by regulating the activation of microglia and inflammation via autophagy modulation.

\section{Methods \\ Animals}

Male C57BL/6 mice weighing 20-30 g were used in this study. All animals were obtained from the Experiment Animal Center of Chongqing Medical University (Chongqing, China). Mice were kept in the standard experimental environment at $22 \pm 2{ }^{\circ} \mathrm{C}$ and $50 \pm 5 \%$ relative humidity with an alternating 12/12-h light/dark cycle. Food and water were provided ad libitum. Before the experiment, all the animals were acclimatized to the environment for at least 1 week and then be randomly assigned to different experimental groups on the basis of 
a random number sequence generated by Excel Software. The detailed procedure is illustrated as follows: (1) mice fulfilling the experimental criteria were selected and numbered. (2) Animal numbers were input into an Excel table, and a random sequence was generated using the "rand" function in Excel software; (3) the animal numbers were rearranged according to the ascending order of random sequence; (4) based on the new order, experimental animals were assigned to receive different treatments, with six mice in each group. Since the model induces pain in animals, the number of mice used was the minimum necessary to achieve sufficient statistical power. Figure $1 \mathrm{~b}$ shows the detailed information about experimental group and sample size of mice for each experiment. The experimental protocol was approved by the Animal Care and Use Committee at Chongqing Medical University in China. All animal procedures were conducted in accordance with the National Institutes of Health Guide for the Care and Use of Laboratory Animals.

\section{Establishment of the chronic migraine model}

The CM model was established as the previously described [29]. Nitroglycerin (NTG) (Beijing Regent, China) was prepared from a stock solution of $5.0 \mathrm{mg} / \mathrm{ml}$ NTG dissolved in 30\% alcohol, 30\% propylene glycol, and water. Before injection, NTG was freshly diluted to $1 \mathrm{mg} / \mathrm{ml}$ with $0.9 \%$ saline. Solution containing $0.9 \%$ saline, $6 \%$ propylene glycol, and $6 \%$ alcohol was used as a vehicle control. The animals intraperitoneally (i.p.) administered of $10 \mathrm{mg} / \mathrm{kg}$ of NTG or an equal volume of vehicle every second day for 9 days (five times in total) (Fig. 1a). All experimental animals were subjected to the behavioral tests described in detail below before and $2 \mathrm{~h}$ after NTG injection (Fig. 1a).

\section{Drug administration}

To explore the level of autophagic flux in CM, $60 \mathrm{mg} / \mathrm{kg}$ chloroquine (CQ, MedChemExpress/MCE, American) was intraperitoneally injected once a day for 9 consecutive days, before NTG treatment and after baseline threshold measurement (Fig. 1a). To investigate the role of autophagy in CM, the autophagy inducer, rapamycin (RAPA, Selleck, TX, USA), was delivered in the same manner at a concentration of $1 \mathrm{mg} / \mathrm{kg}$ (Fig. 1a). The P2X7R selective antagonist, Brilliant Blue G (BBG, Sigma-Aldrich, Hungary), was used to figure out the function of P2X7R in CM. BBG was intraperitoneally administered at a dose of $50 \mathrm{mg} / \mathrm{kg}$ every other day for 9 days, in an identical manner to NTG treatment (Fig. 1a). CQ and BBG were diluted in $0.9 \%$ saline. The RAPA was dissolved in 2\% DMSO, 30\% PEG 300, and 5\% Tween 80. An equivalent volume of solvent corresponding to each drug was used as a vehicle control. The drug dosage and methods of delivery were determined by literature data and preliminary experiments $[16,30,31]$. All drug solutions were freshly prepared on the day of use.

\section{Behavioral assessment}

In the clinic, central sensitization is manifested as cutaneous allodynia and expansion of the pain area, including the craniofacial and non-craniofacial region [32]. Therefore, in the animal model, we measured both the periorbital and hind paw withdrawal threshold to mechanical stimulation, as performed in the previous references. The plantar thermal sensitivity was also

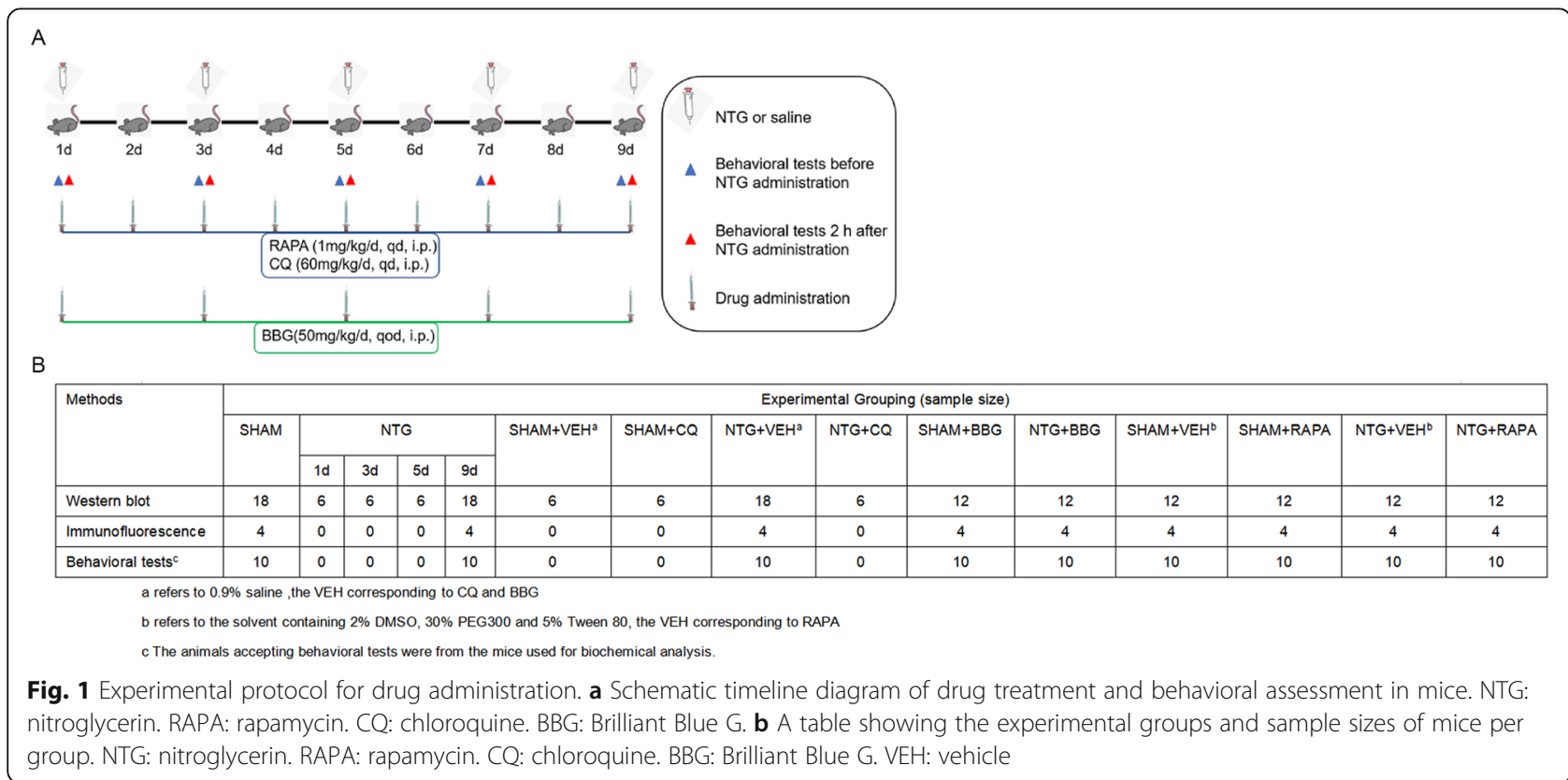


evaluated, since chronic NTG delivery induces thermal hyperalgesia [33]. All the behavioral assessments were performed between 9:00 am to 15:00 pm in a quiet environment. Mice underwent a 3-day training period before the experiment. All the behavioral tests including mechanical withdrawal threshold and thermal withdrawal latency were performed in the same sets of animals. At the end of the experiment, six mice with successful modeling and significant behavioral tests were selected for further biochemical and morphological analyses. The behavioral testing was performed by the same investigators, who were blinded to the treatment groups.

\section{Measurement of the mechanical withdrawal threshold}

The mechanical withdrawal threshold of the hind paw and periorbit was assessed every other day before and 2 $\mathrm{h}$ after NTG injection (Fig. 1a). We used Von Frey filaments with the up-down method to determine the withdrawal threshold to mechanical stimulation as previously described [7]. Briefly, a series of Von Frey filaments (range from $0.01 \mathrm{~g}$ to $2 \mathrm{~g}$ ) were applied to the hind paw or periorbit, with an initial stimulation strength of $0.4 \mathrm{~g}$. If there was no response to the stimulation, the filament strength was increased; otherwise, the filament strength was decreased until there was a positive reaction. Each filament was held for $5 \mathrm{~s}$ at the testing site with an interval of $1 \mathrm{~min}$. The threshold was recorded as the lowest force evoking a positive response and averaged from three repetitive measurements.

For the hind paw test, the mice were separately placed in a suspended acrylic chamber covered with a wire mesh floor. The animals were acclimated to the new environment for at least $30 \mathrm{~min}$. The Von Frey filaments were applied perpendicularly to the central area of the hind paw surface. Brisk withdrawal, shaking, lifting, or licking of the testing paw were considered positive responses. For the periorbital assessment, the mice were placed individually in a 4-oz. paper cup, allowing only the head to poke out. The head and fore paw could move freely, but body could not turn in the cup. The periorbital region included the area from caudal of eyes to approximately the midline. Vocalization, quick retraction of head from the stimulation, or scratching of face with the ipsilateral fore paw were considered positive responses.

\section{Measurement of thermal withdrawal latency}

The thermal withdrawal latency of the hind paw was assessed every other day before and $2 \mathrm{~h}$ after NTG injection (Fig. 1a). We used a plantar test apparatus (Techman PL-200, Chengdu, China) with an intensity adjustable radiant heat to assess the thermal sensitivity. Briefly, mice were placed separately in a transparent chamber with a temperature controlled glass floor. After
30 min of acclimatization, the radiant thermal stimulus was delivered to the central part of the hind paw through the glass. The stimulus was shut off once the hind paw moved and the thermal withdrawal latencies were recorded automatically. The radiant heat intensity was calibrated to produce a basal withdrawal latency of $8-10 \mathrm{~s}$ in the control group. The cut-off time was $20 \mathrm{~s}$ to prevent tissue damage [33]. The radiant heat was delivered three times to each hind paw with an interval of $5 \mathrm{~min}$. The thermal withdrawal latencies were defined as the average of three recordings.

\section{Western blot analysis}

Mice were sacrificed under anesthesia with $10 \%$ chloral hydrate. The TNC was collected immediately and stored at $80{ }^{\circ} \mathrm{C}$. Tissues were homogenized in cold RIPA lysis buffer (Beyotime, Shanghai, China) containing a protease inhibitor, phenylmethylsulphonyl fluoride (PMSF, Beyotime, Shanghai, China) at $4{ }^{\circ} \mathrm{C}$ for $1 \mathrm{~h}$. The lysate was centrifuged at $12000 \times g$ for $15 \mathrm{~min}$ in $4{ }^{\circ} \mathrm{C}$, and then the protein concentration of the supernatant was determined with a BCA protein assay kit (Beyotime, Shanghai, China). The protein was denatured by heating at $100{ }^{\circ} \mathrm{C}$ for $5 \mathrm{~min}$ and stored at - $80{ }^{\circ} \mathrm{C}$. Equal amounts of tissue protein (40 ug) were separated on $10 \%$ or $12 \%$ sodium dodecyl sulfatepolyacrylamide (SDS-PAGE) gels (Beyotime, Shanghai, China), and electrotransferred to polyvinylidene difluoride (PVDF) membranes (Millipore, USA). The membranes were blocked in Tris-buffered saline with Tween-20 (TBST buffer) containing 5\% non-fat milk for $2 \mathrm{~h}$ at room temperature and incubated overnight at $4{ }^{\circ} \mathrm{C}$ with the following primary antibodies: rabbit anti-P2X7R, rabbit anti-CGRP, mouse anti-c-fos, rabbit anti-LC3, rabbit antiSQSTM1/p62, rabbit anti-beclin1, rat anti-NLRP3, rabbit anti-IL-1 $\beta$, rabbit anti-IL- 18 , and mouse anti- $\beta$-actin. The detailed information for all the antibodies used in this study is provided in Table 1 . All the primary antibodies were diluted with special diluent (Beyotime, Shanghai, China). The next day, the membranes were washed three times with TBST for $10 \mathrm{~min}$ each and incubated with corresponding horseradish peroxidase conjugated secondary antibodies, including goat-anti-rabbit, goat-anti-mouse and goat-anti-rat, for $1 \mathrm{~h}$ at room temperature. The immunoreacted bands were revealed with an ECL detection kit (Advansta Inc., USA), visualized and analyzed with an imaging system (Fusion, Germany). $\beta$-actin was used to normalize the relative expression of the target proteins in different groups. Each western blot was repeated at least six times, and the consistent results were obtained.

\section{Immunofluorescence staining}

To detect c-fos, tissues were collected $2 \mathrm{~h}$ after the last NTG or vehicle injection, while for other targets, tissues were collected within $12 \mathrm{~h}$. Mice were anesthetized with 
Table 1 Antibody used in western blotting analysis, immunofluorescence staining assays

\begin{tabular}{|c|c|c|c|c|}
\hline Antibody & Manufacturer & Catalog number & Host & Dilution \\
\hline \multicolumn{5}{|l|}{ For western blot analysis } \\
\hline $\mathrm{P} 2 \times 7$ & Sigma-Aldrich, USA & P8232 & Rabbit & $1: 3000$ \\
\hline LC3 & Bimake, USA & A5202 & Rabbit & $1: 500$ \\
\hline Beclin1 & Cell signaling technology, USA & 3495 & Rabbit & $1: 1000$ \\
\hline P62 & Cell signaling technology, USA & $5114 \mathrm{~T}$ & Rabbit & $1: 1000$ \\
\hline NLRP3 & Abcam, UK & ab205680 & Rat & $1: 1000$ \\
\hline $\mathrm{IL}-1 \beta$ & Bioss, China & bs-6319R & Rabbit & $1: 1000$ \\
\hline IL-18 & Abcam, UK & ab207323 & Rabbit & $1: 1000$ \\
\hline CGRP & Abcam, UK & Ab139264 & Rabbit & $1: 3000$ \\
\hline$c-f o s$ & Santa Cruz, USA & sc-447 & Mouse & $1: 1500$ \\
\hline$\beta$-actin & ZSGB-BIO, China & TA-09 & Mouse & $1: 3000$ \\
\hline $\mathrm{HRP}^{\mathrm{a}}$ conjugated anti-rabbit & ZSGB-BIO, China & ZB-2301 & Goat & $1: 9000$ \\
\hline HRP conjugated anti-mouse & ZSGB-BIO, China & ZB-2305 & Goat & $1: 5000$ \\
\hline HRP conjugated anti-rat & ZSGB-BIO, China & ZB-2307 & Goat & $1: 5000$ \\
\hline \multicolumn{5}{|l|}{ For immunofluorescence staining } \\
\hline $\mathrm{P} 2 \times 7$ & Sigma-Aldrich, USA & P8232 & Rabbit & $1: 500$ \\
\hline CGRP & Santa Cruz, USA & sc-57053 & Mouse & $1: 100$ \\
\hline c-fos & Novus Biologicals, USA & NBP2-50057SS & Rabbit & $1: 5000$ \\
\hline Iba1 & Wako, Japan & 019-19741 & Rabbit & $1: 800$ \\
\hline lba1 & Novus Biologicals, USA & NB100-1028SS & Goat & $1: 800$ \\
\hline GFAP & Santa Cruz, USA & sc-33673 & Mouse & $1: 200$ \\
\hline NeuN & Abcam, UK & ab104224 & Mouse & $1: 500$ \\
\hline Cy3 Goat anti-rabbit IgG & Beyotime, China & A0516 & Goat & $1: 500$ \\
\hline Alexa Fluor 488 Goat anti-mouse lgG & Beyotime, China & A0428 & Goat & $1: 500$ \\
\hline Alexa Fluor 555 Donkey anti-rabbit lgG & Beyotime, China & A0453 & Donkey & $1: 500$ \\
\hline IFkine Green, Donkey anti-goat lgG & Abbkine, USA & A-24231-1 & Donkey & $1: 400$ \\
\hline
\end{tabular}

a HRP horseradish peroxidase

$10 \%$ chloral hydrate and perfused transcardially with 60 $\mathrm{ml}$ of cold phosphate-buffered saline (PBS, pH 7.4) followed by $60 \mathrm{ml}$ of $4 \%$ cold paraformaldehyde (PFA) in $0.1 \%$ PBS ( $\mathrm{pH} 7.4$ ). The brainstem and cervical spinal cord $(\mathrm{C} 1-\mathrm{C} 2)$ were harvested and postfixed in $4 \%$ PFA for $24 \mathrm{~h}$ at $4{ }^{\circ} \mathrm{C}$. The medullary segment containing the TNC between +1 and $-3 \mathrm{~mm}$ from the obex was removed and dehydrated sequentially in 20\% and 30\% sucrose until the tissue sank. Tissues were embedded, flash frozen with 2-methylbutane (Aladdin, Shanghai, China) and sliced coronally into 10 um sections with a cryostat (Leica, Japan). After antigen retrieval with sodium citrate (Beyotime, Shanghai, China), the sections were blocked and permeabilized simultaneously with 0.3\% Triton X-100 (Beyotime, Shanghai, China) in 5\% donkey or goat serum (Boster, Wuhan, China) for 30 minutes at $37{ }^{\circ} \mathrm{C}$. Then, the sections were incubated overnight at $4{ }^{\circ} \mathrm{C}$ with the following primary antibodies: rabbit anti-P2X7R, mouse anti-CGRP, rabbit anti-c-fos, rabbit anti-Iba1, goat anti-Iba1, mouse anti-NeuN, and mouse anti-GFAP. The detailed information for all the antibodies is provided in Table 1 . All the primary antibodies were diluted with blocking solution. After rinsing three times for $15 \mathrm{~min}$ in PBS, the sections were reacted with corresponding secondary antibodies (conjugated to Alexa Fluor 488, 555 or cy3) for $90 \mathrm{~min}$ at $37^{\circ} \mathrm{C}$. Nuclei were stained with 4',6-diamidino-2-phenylinodole (DAPI) (Beyotime, Shanghai, China) for $10 \mathrm{~min}$ at $37{ }^{\circ} \mathrm{C}$. Images were captured with a confocal microscope (LSM800, ZEISS, Germany). Negative control sections were treated with PBS instead of primary antibody and showed no positive signals.

\section{Immunofluorescence imaging data analysis}

The TNC area was determined based on the morphology under a light microscope according to the Mouse Brain Atlas [34]. The mean optical density of CGRP was analyzed using ImageJ software (version 1.8.0_112) with a $\times 10$ objective. To quantify the number of immunopositive cells of Iba1 and c-fos, the squared images (field of 
view, FOV, $320 \times 320 \mathrm{um}^{2}$ ) in the superficial layer of the TNC (Fig. 4f) were taken at $\times 200$ magnification. Four to six FOV per section were investigated. ImageJ software (version 1.8.0_112) was used to count the immunoreactive cells. To analyze microglial morphology, Neuron J, an ImageJ plug-in, was used to determine the total and mean length of microglial processes. Each cohort consisted of 4 mice, and 6-8 sections from each mouse were analyzed. The image collection and analysis were performed by an experimenter who was blinded to the treatment groups.

\section{Statistical analysis}

GraphPad Prism version 7.0 (GraphPad Software Inc., San Diego, CA, USA) was used for the statistical analysis and graph generation. All the data are presented as the mean \pm standard error of the mean (SEM). The Kolmogorov-Smirnov test and Bartlett's tests were used to analyze the normality and homogeneity of the data, respectively. Differences between two groups were determined by an independent-sample $t$ test. Comparison among three or more groups were investigated by oneway analysis of variance (ANOVA) followed by Tukey's or Dunnett's multiple comparison tests to detect pairwise between-group differences. Behavioral data were analyzed by two-way ANOVA with the Bonferroni post hoc test. Two-tailed tests were applied in all statistical analyses. $P<0.05$ was considered to be statistically significant.

\section{Results}

Autophagic flux impairment in the TNC after chronic NTG administration

LC3-II is expressed in the autophagosome membrane and is widely used as a reliable autophagy marker since the protein level of LC3-II strictly reflects the number of autophagosomes [35]. The accumulation of LC3-II is usually caused by increased autophagosome formation due to enhanced autophagic flux or dysfunctional degradation of the autophagosome due to impaired autophagic flux [22, 28, 35]. The p62 protein is a well-known autophagy substrate that helps to determine the reason for the change in LC3-II, thus providing the additional evidence of autophagic flux [35]. The activated autophagy usually causes a decrease in p62, while the inhibited autophagy leads to a complete opposite effect [35, 36]. In addition, beclin1 is also a key protein reflecting the autophagy activity [35]. Therefore, we detected the expression of autophagy-related proteins, including LC3-II, beclin1, and p62, to evaluate the level of autophagic flux in the CM model.

As shown in Fig. 2, recurrent NTG treatment significantly increased the ration of LC3-II/LC3-I, augmented the expression of p62, and did not alter the level of beclin1, which indicated an impaired autophagic flux (Fig. $2 \mathrm{a}-\mathrm{c}$ ). To further confirm the change in autophagic flux, we intraperitoneally administered CQ to inhibit lysosome function by increasing the $\mathrm{PH}$ of the lysosomal cavity. The data showed that, compared with mice receiving NTG only, the combination treatment of CQ and NTG did not further increase the ratio of LC3-II/ LC3-I (Fig. 2d). The expression of p62 had an increasing trend, but the difference was not statistically significant (Fig. 2e). The level of beclin1 was similar in mice treated with CQ and those without CQ (Fig. 2f). These combined data revealed an impaired autophagic flux in the TNC of the CM model caused by a dysfunctional degradation pathway of autophagy, thus leading to the upregulation of autophagy-related structural proteins.

\section{Autophagy induction prevented basal rather than acute mechanical and thermal hyperalgesia}

In line with previous studies, chronic NTG injection induced a gradual decrease in the basal pain threshold over time, and each single NTG treatment produced an acute reduction of the pain threshold after $2 \mathrm{~h}$. To determine the role of autophagy in CM, we used RAPA, an autophagy inducer, to activate autophagy and evaluated the effect of RAPA on NTG-induced hyperalgesia. The RAPA (NTG-RAPA) markedly attenuated the basal mechanical and thermal hyperalgesia, while it had no effect on the post-treatment response (Fig. 3). Only RAPA treatment (SHAM-RAPA) did not alter the pain threshold. These data suggested that autophagy in the TNC exerted a beneficial effect against CM development. The autophagy activation contributed greatly to the reduction of CM-liked pain behavior.

\section{Activation of autophagy reduced CGRP and c-fos} expression in the TNC after recurrent NTG administration To investigate the effect of autophagy on central sensitization, mice were injected intraperitoneally with RAPA ( $1 \mathrm{mg} / \mathrm{kg} /$ day) to activate autophagy, once a day for 9 days. We found the up-regulated ration of LC3-II/ LC3I in combination with decreased expression of p62 and an elevated protein level of beclin 1 in mice with RAPA treatment, compared with mice administered NTG only (Fig. 4a-c). The expression changes in autophagy-related proteins are consistent with characteristics of activated autophagy. No difference was found in P2X7R expression between modeling mice treated with and without RAPA (Fig. 4d), which indicated that autophagy acted downstream of P2X7R in the TNC. CGRP is a pivotal endogenous neuropeptide involved in the initiation and development of CM. A large number of studies have shown that CGRP plays an important role in central sensitization [37, 38]. Western blot showed that the protein level of CGRP in mice pretreated 


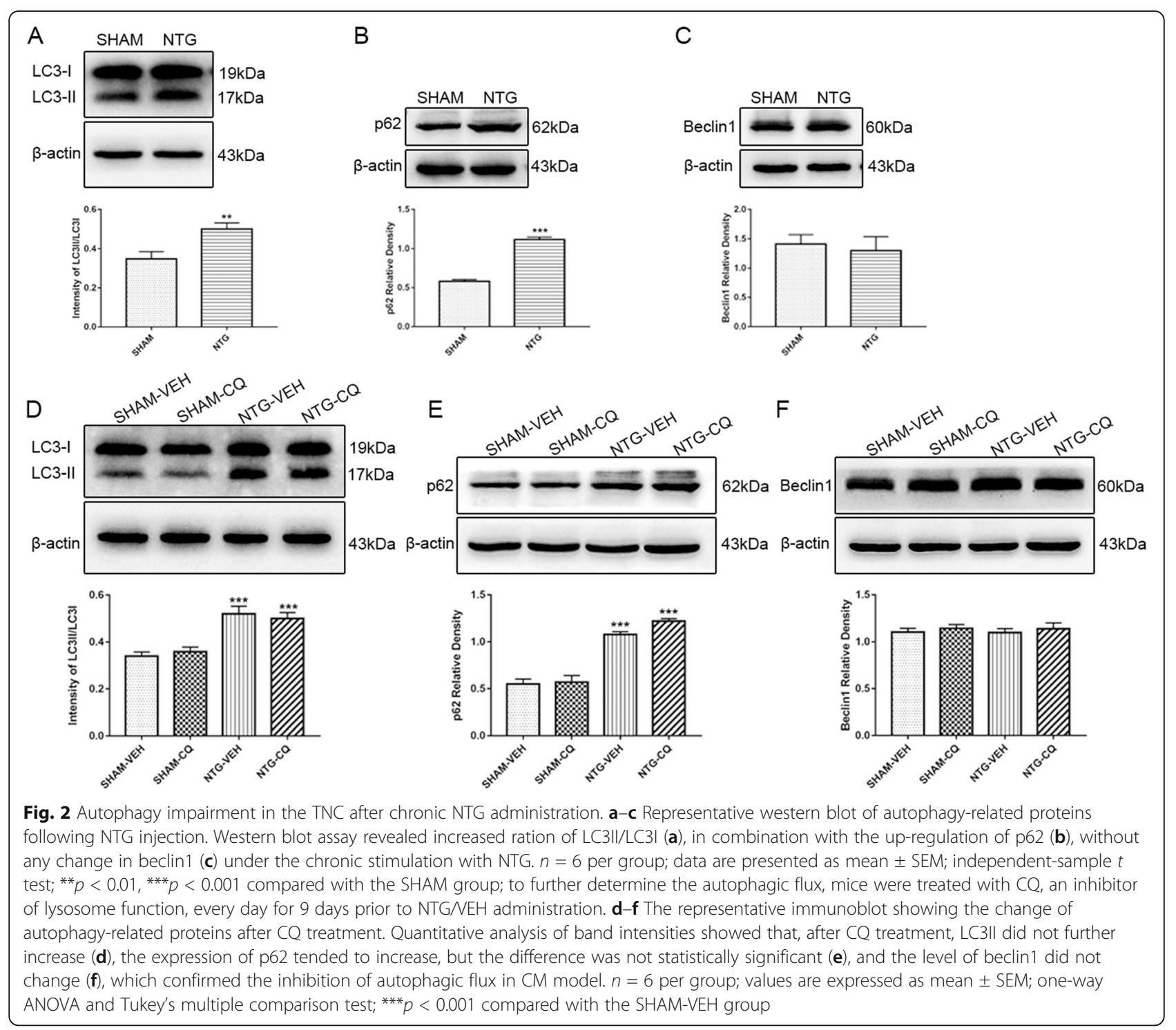

with RAPA was lower than that in mice treated with NTG (Fig. 4e). The mean optic density (OD) of CGRP immunoreactive fibers in the superficial layers of the TNC was also reduced by RAPA delivery (Fig. 4g, h). c-fos is recognized as a reliable marker of neuronal activation to nociceptive stimulation. The increased expression of $\mathrm{c}$-fos in the trigeminal pain pathway suggests the activation of nociceptive neurons, which is closely related to central sensitization [39]. Consistent with our previous studies, the protein level of c-fos was upregulated following NTG injection (Fig. 4e). The number of c-fos-positive cells in the superficial layer of the TNC also increased (Fig. 4i, j). Pretreatment with RAPA attenuated the NTG-induced expression of c-fos both in western blot grayscale analysis and immunofluorescence quantitative analysis (Fig. 4e, $i, j)$. These combined data suggested that the activated autophagy alleviated central sensitization of CM.

\section{Autophagy induction attenuated the NTG-evoked} activation of microglia and NLRP3 inflammasome

Previous studies have shown that autophagy regulates the activation of microglia and inflammatory response $[40,41]$. The activation of NLRP3 inflammasome is inhibited by autophagy through the clearance of damaged mitochondria and degradation of the NLRP3 inflammasome [26]. Therefore, we used western blot analysis to detect the expression of NLRP3 and related inflammatory factors, IL-18 and IL-1 $\beta$. Immunofluorescence staining was used to analyze changes in microglial quantity, immunoreactivity, and morphology. Consistent with our previous studies, NTG treatment significantly increased the expression of NLRP3, IL-18, and IL-1 $\beta$ in the TNC (Fig. 5a-d). Immunofluorescence staining of Iba1 showed that the number of Iba1-positive cells increased with enhanced immunoreactivity after NTG 


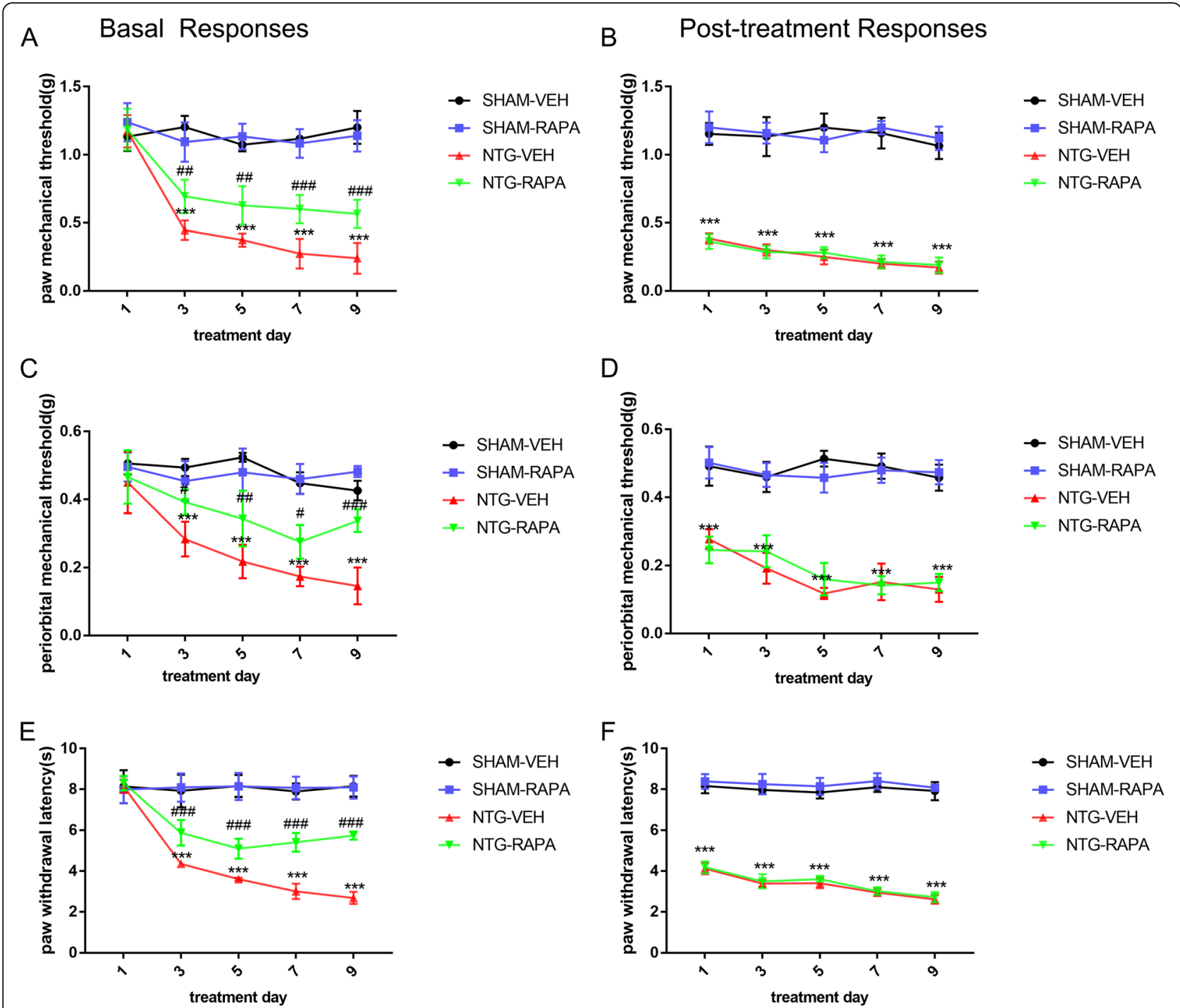

Fig. 3 Autophagy activation attenuated NTG-induced basal, not acute, mechanical, and thermal hyperalgesia. a, c, e Repeated administration of RAPA, the autophagy inducer, improved basal mechanical hyperalgesia of the hind paw (a), periorbit (c), and thermal hyperalgesia of the hind paw (e). $\mathbf{b}, \mathbf{d}$, $\mathbf{f}$ RAPA did not prevent the post-treatment responses comprising hind paw mechanical hypersensitivity (b), periorbital mechanical hypersensitivity (d), and thermal hyperalgesia (f). $n=10$ per group; data are presented as the mean \pm SEM; two-way ANOVA and Bonferroni post hoc test were

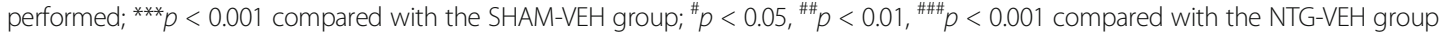

administration (Fig. 5e-h). Morphologically, the microglia presented with hypertrophied cell bodies and shorter and fewer processes. The quantitative analysis showed that the total and mean length of processes decreased (Fig. 5e, f, i ,j). Mice receiving RAPA treatment exhibited a significant decrease in the expression levels of NLRP3, IL-18, and IL-1 $\beta$, compared to mice receiving NTG only (Fig. 5a-d). In addition, RAPA pretreatment inhibited microgliosis, reduced Iba1 immunoreactivity, and changed the microglia into a ramified shape, exhibiting increases in the total and mean length of processes (Fig. $5 \mathrm{e}-\mathrm{j}$ ). Taken together, these results revealed that enhanced autophagic flux suppressed the activation of microglia and the NLRP3 inflammasome.

\section{P2X7R was increasingly expressed in the TNC area after recurrent NTG administration}

To investigate the effect of NTG treatment on P2X7R expression, we monitored the protein level of P2X7R in the TNC at different time points during modeling. First, as shown in Fig. 6a, c, the expression of CGRP increased gradually with the development of modeling, which was consistent with the hyperalgesia induced by NTG and suggested a successful CM model. The expression level 


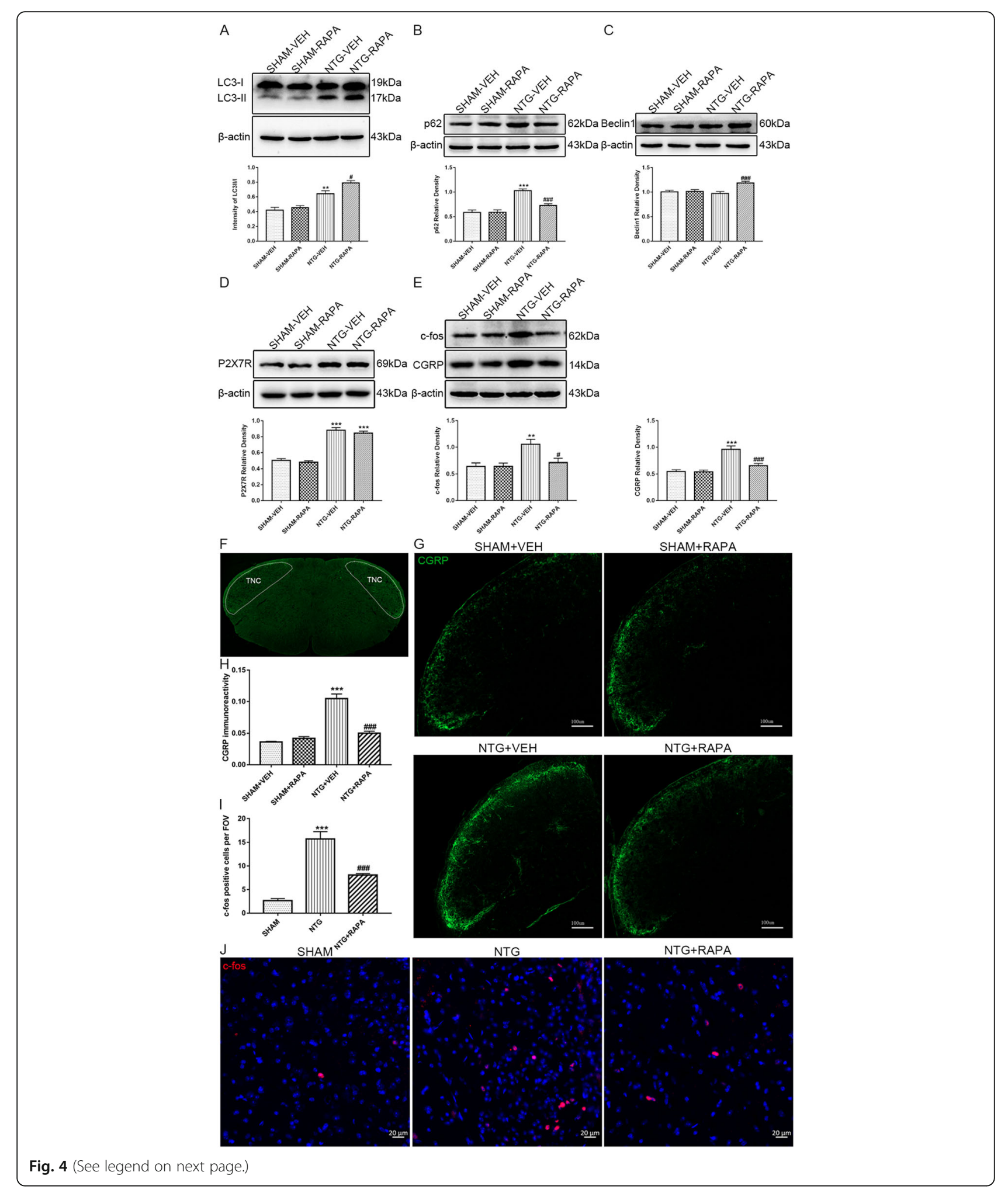




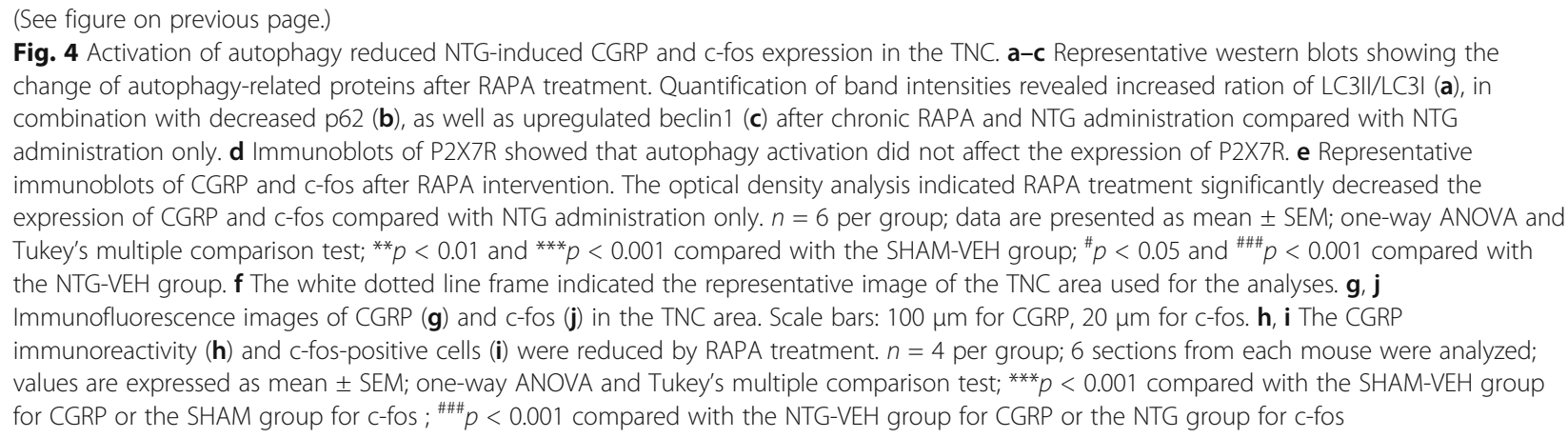

of P2X7R continuously rose in a time-dependent manner following repeated intermittent NTG injection. Specifically, the P2X7R protein level significantly increased on the third day and peaked on the last day of NTG treatment (Fig. 6a, b). To determine the cell types expressing P2X7R in the TNC, we performed double immunofluorescence staining to label the P2X7R protein and cell type-specific markers, including Ibal-1 for microglia, NeuN for neurons, and GFAP for astrocytes. The images showed that P2X7R was mainly co-localized with Iba1, but not with NeuN or GFAP (Fig. 6d). The results indicated that $\mathrm{P} 2 \mathrm{X} 7 \mathrm{R}$ was mainly expressed in microglia. Collectively, these data revealed that recurrent NTG administration provoked a significant increase in microglial P2X7R in the TNC.

\section{Blockage of P2X7R partially prevented mechanical and thermal hyperalgesia}

To determine whether P2X7R contributed to the development of $\mathrm{CM}$, mice received an intraperitoneal injection of the specific P2X7R antagonist, BBG $(50 \mathrm{mg} / \mathrm{kg}$ ), prior to NTG injection every other day for 9 days. We found that BBG treatment notably increased the mechanical withdrawal threshold of the hind paw and periorbit, and prolonged the thermal withdrawal latency of the hind paw. In addition, BBG was effective in ameliorating both the basal and acute hyperalgesia (Fig. 7). Therefore, we concluded that P2X7R was involved in the initiation and development of CM. Blockage of P2X7R had a positive effect on alleviating $\mathrm{CM}$-associated pain.

\section{P2X7R inhibition decreased CGRP and c-fos expression in} the TNC after repetitive intermittent NTG treatment

To determine whether the upregulation of P2X7R contributed to central sensitization of CM, we delivered BBG and evaluated its effect on the expression of CGRP and c-fos. As shown by western blot analysis, we detected a remarkable decrease in CGRP and c-fos in the TNC homogenized tissue after P2X7R inhibition (Fig. 8b). Immunofluorescence staining showed that the immunoreactive intensity of CGRP fibers and the number of c-fos-positive cells were significantly reduced following BBG treatment, which was consistent with the results of western bolt analysis (Fig. 8c-f). These data supported the contribution of P2X7R to the pathophysiological mechanism of CM.

\section{Blockage of P2X7R activated autophagic flux in the TNC}

Numerous studies have indicated the involvement role of P2X7R in autophagy regulation [20, 23]. Short-term P2X7R activation has been shown to stimulate autophagic flux, while persistent P2X7R stimulation leads to autophagic dysfunction [22]. Here, we assessed the effect of P2X7R on autophagic flux in the CM. The western blot analysis suggested that BBG treatment significantly decreased the LC3-II/LC3-I ratio and reduced the expression of p62. The protein level of beclin1 was similar between mice receiving both BBG and NTG and mice receiving NTG only (Fig. 9a-c). The results indicated that blocking P2X7R could enhance the level of autophagic flux in the TNC.

\section{P2X7R inhibition reduced the activation of microglia and the NLRP3 inflammasome}

To elucidate the role of $\mathrm{P} 2 \mathrm{X7R}$ in the regulation of microglia and NLRP3 inflammasome activation, we measured the expression of NLRP3 and related inflammatory factors using western blotting. Immunofluorescence was applied to detect the change in quantity and morphology of the microglia. We found that the levels of NLRP3, IL$1 \beta$, and IL-18 in mice receiving P2X7R antagonist were significantly lower than that in mice without P2X7R inhibition (Fig. 10a-d). After chronic pretreatment with BBG, both the Iba1 immunoreactivity and the number of positive cells were reduced compared with NTG group (Fig. 10e-h). Additionally, the total and mean length of the microglial processes were notably increased (Fig. 10e, f, i, j). Morphologically, BBG treatment changed the microglia into a ramified shape, which was similar to that in the SHAM group (Fig. 10e, f). Therefore, we concluded that P2X7R played a pivotal role in microglia and NLRP3 inflammasome activation. 


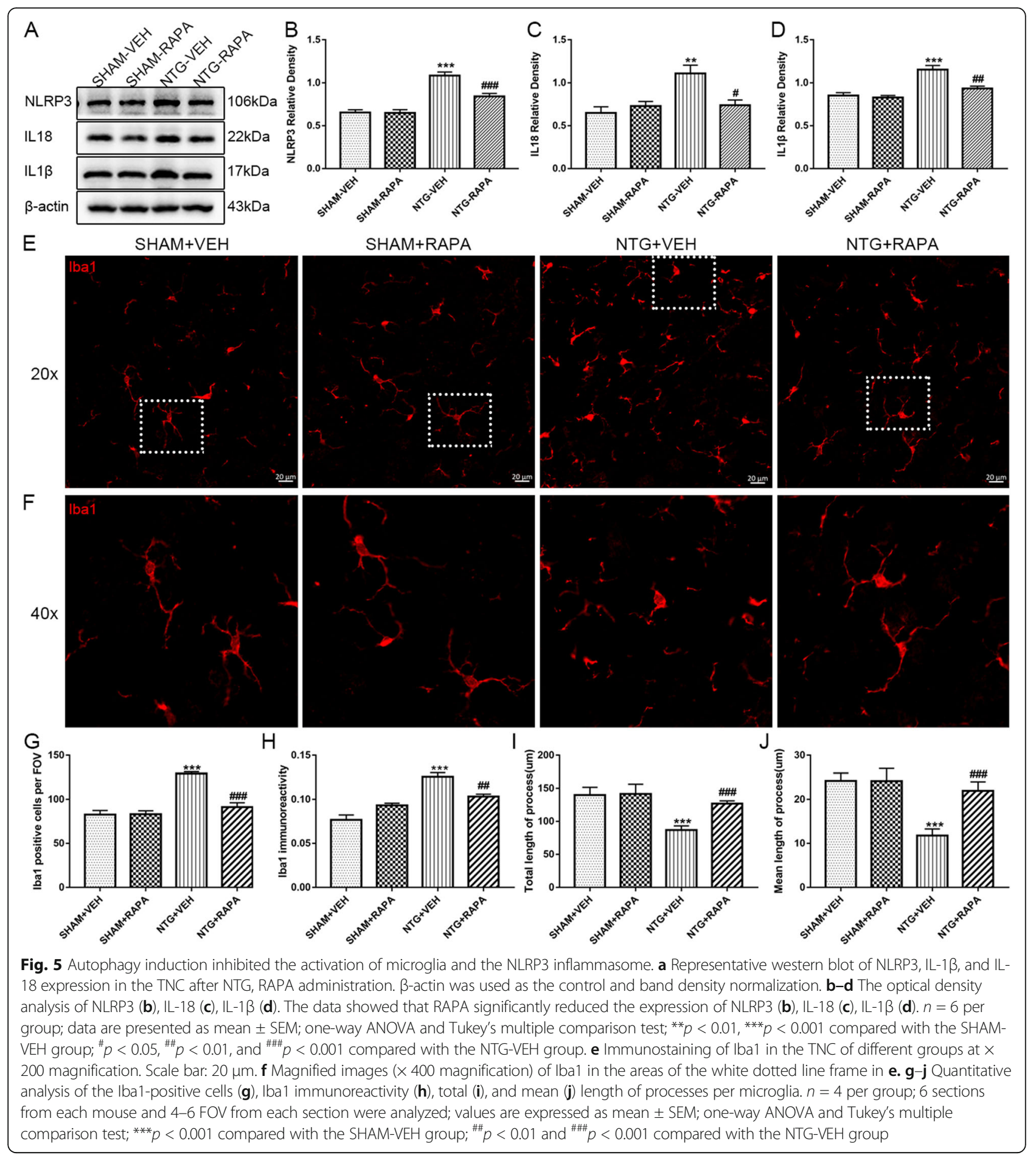

\section{Discussion}

Our current study provided several new findings as follows. First, we observed dysfunctional autophagic flux in the NTG-induced CM model and determined the beneficial effect of autophagy activation on pain relief for the first time. Subsequently, we confirmed that microglial P2X7R in the TNC was involved in the pathophysiological process of CM. In addition, we provided the first evidence from the in vivo experiment of CM that P2X7R activation promoted the activation of microglia and the NLRP3 inflammasome by negatively regulating the autophagic flux, thus contributing to the central sensitization. Our data demonstrated the regulation of autophagy as a novel mechanism by which 


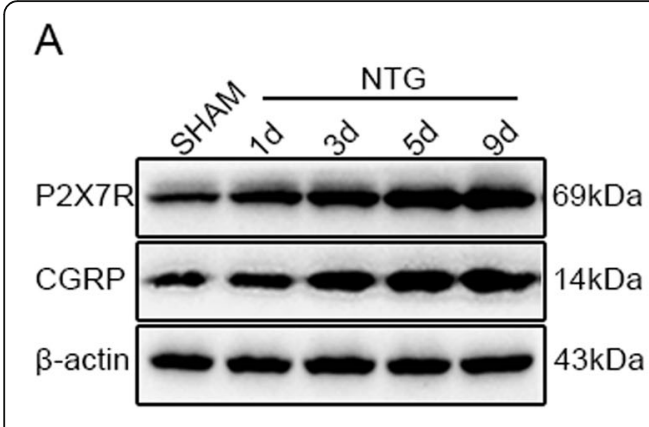

B
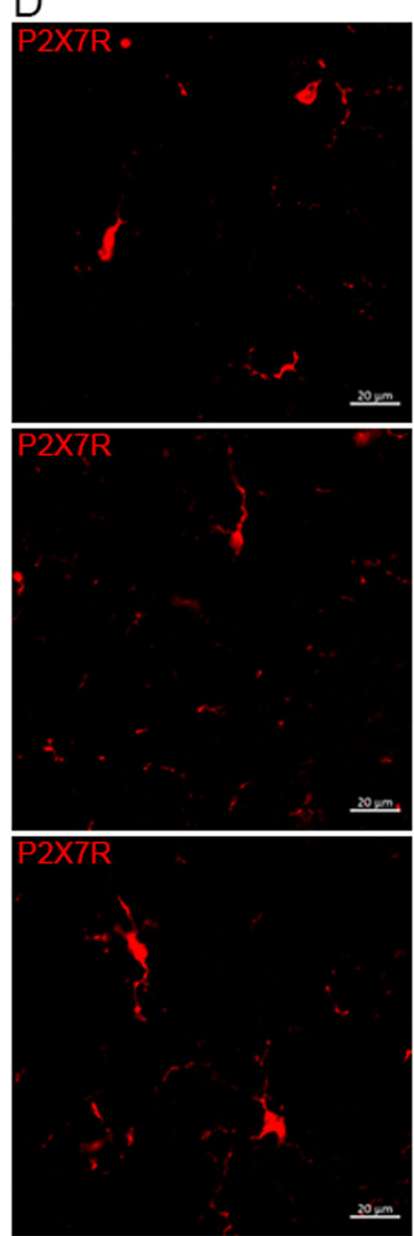
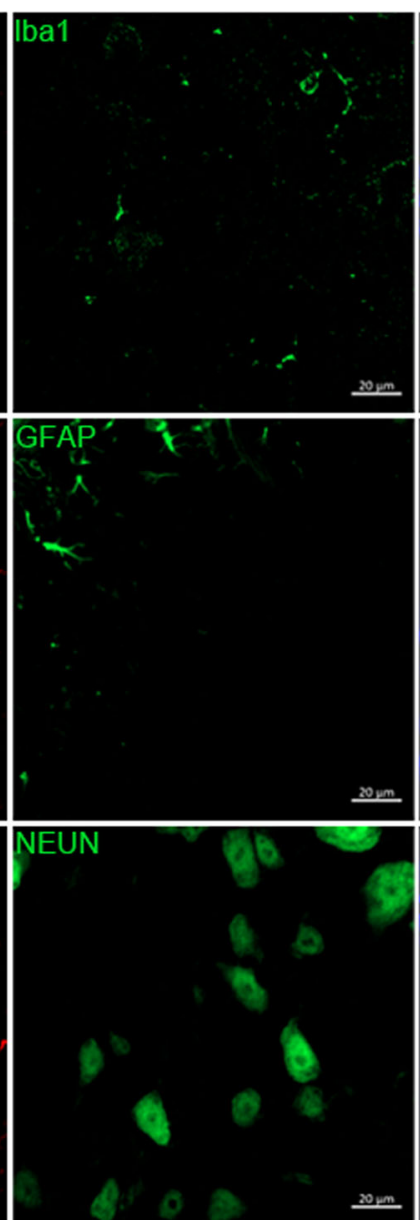

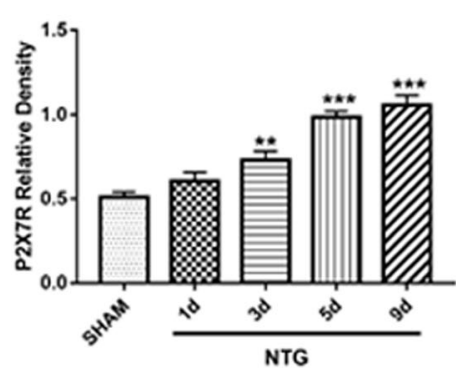

C
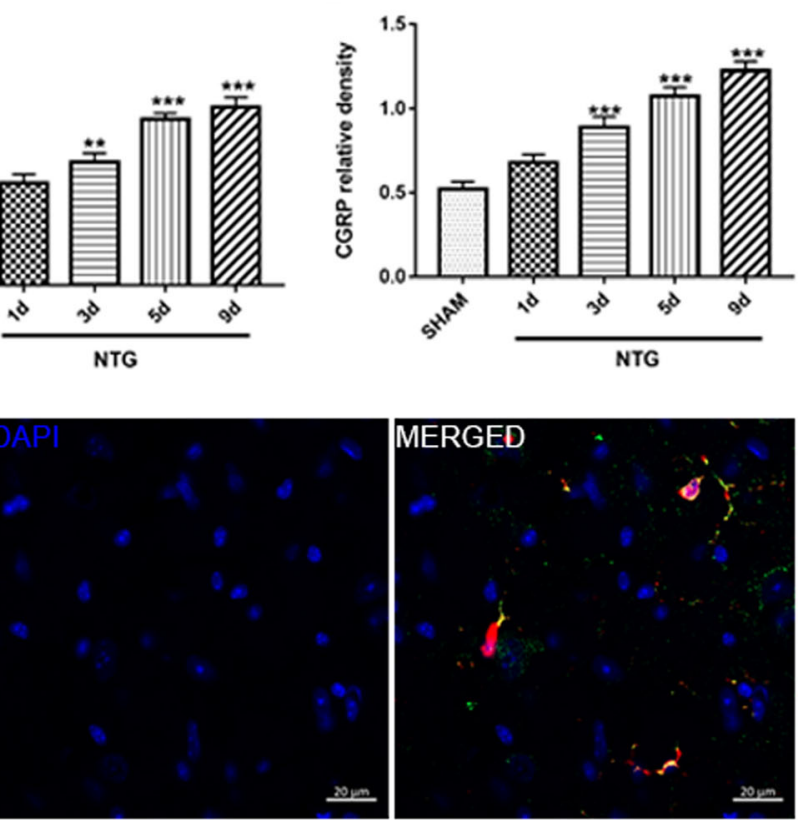

MERGED
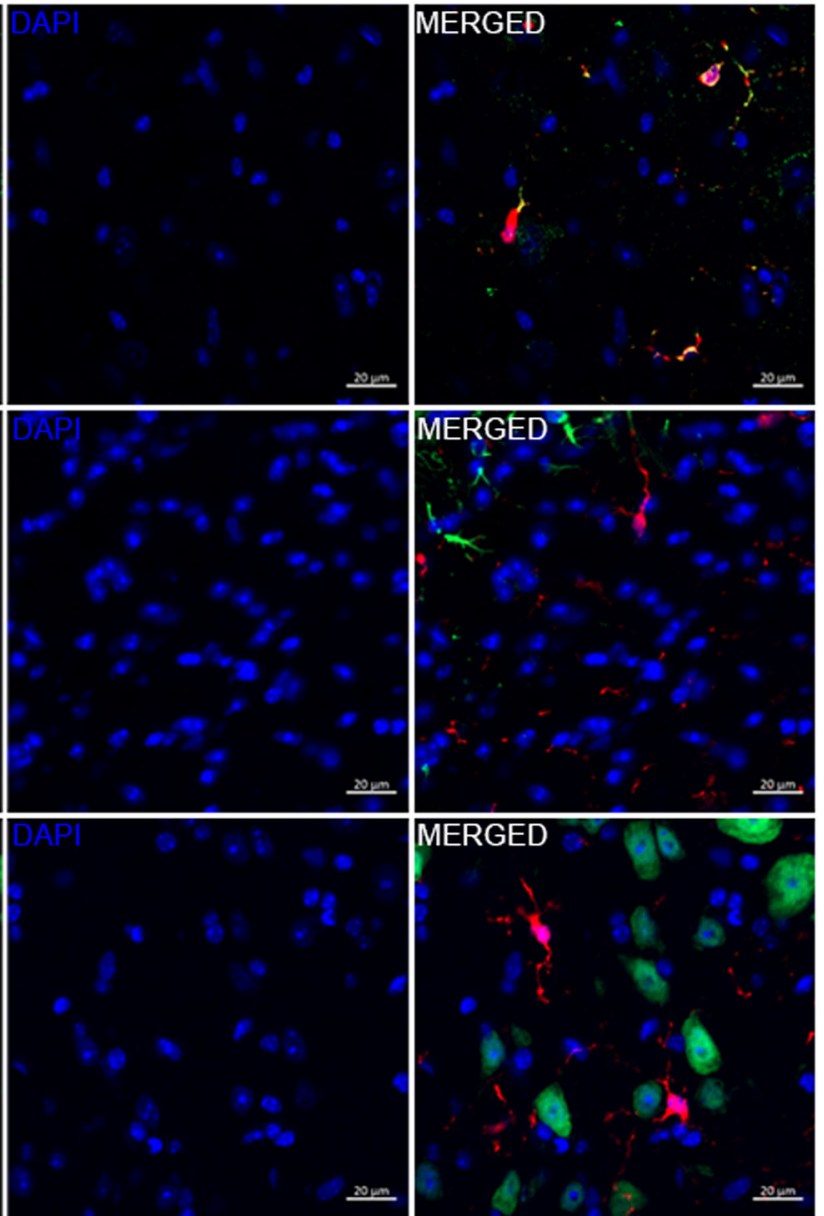

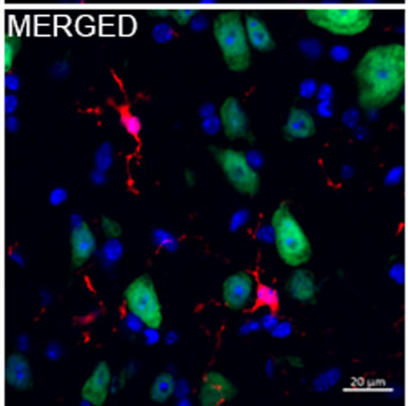

Fig. 6 The microglial P2X7R increased in the TNC after chronic administration of NTG. a Representative western blot of P2X7R and CGRP on different time points in the TNC. $\mathbf{b}, \mathbf{c}$ The band intensities of P2X7R and CGRP relative to those of $\beta$-actin indicated that the protein level of P2X7R (b) and CGRP (c) increased gradually with the development of CM model. $n=6$ per group; data are presented as mean \pm SEM; one-way ANOVA and Dunnett's multiple comparison test; ${ }^{* *} p<0.01$ and ${ }^{* *} p<0.001$ compared with the SHAM group. d Double immunofluorescence staining of P2X7R and Iba1, GFAP, and NEUN in the TNC area. Most P2X7R-positive cells are double labeled with Iba1. Scale bar: $20 \mu \mathrm{m}$

P2X7R modulate the activation of microglia and the NLRP3 inflammasome.

In this study, we used repeated intermittent NTG injection to establish the CM animal model, which is now generally accepted as a reliable mice model of $\mathrm{CM}$ due to the similar features between mice with NTG injection and patients with CM [29]. First, NTG is a definite migraine trigger and is widely used in clinical trials $[42,43]$. Studies have shown that a single treatment of NTG induces a significant decrease in the acute mechanical withdrawal threshold, while repeated injection of NTG evokes a progressive decrease in the basal mechanical withdrawal threshold that persists for 1 week after NTG cessation [43]. This phenomenon mimics the occurrence of cutaneous 


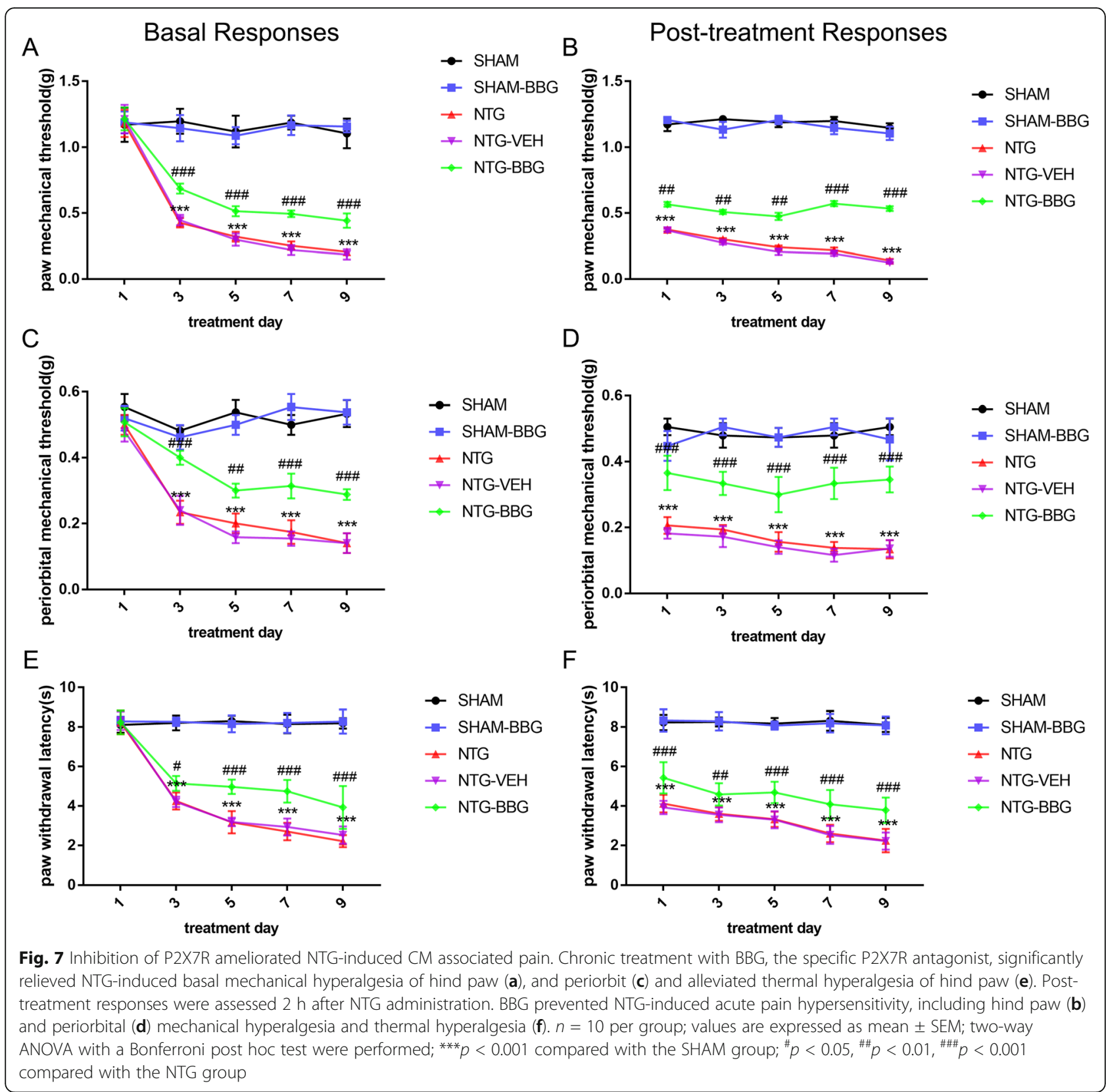

allodynia both in the ictal and interictal period of migraine in CM patients [44]. Additionally, the NTG-induced hyperalgesia can be relieved by the migraine specific analgesic, sumatriptan $[33,42]$. The pain hypersensitivity in the periorbit and hind paw produced by NTG injection corresponds to the cutaneous allodynia of the cephalic and extracephalic region in patients with CM [32]. Furthermore, NTG treatment also elicits some migraine-specific symptoms in mice, comprising photophobia, hypoactivity, and facial grimace behaviors, which are similar to the characteristics of photophobia and activities aggravating headache in patients with CM $[45,46]$. Migraine is more prevalent in women than in men, and estrogen plays an important role in that sex difference $[47,48]$. Numerous studies have shown that the level and stability of estrogen can modulate the transmission of pain pathways $[49,50]$. To avoid the interference of estrogen, the male mice were selected to establish the model, since mice of both sexes can be used to successfully establish the NTG-induced CM model [29]. Given the notable sex differences in migraine prevalence, the sex dimorphism of P2X7R involvement in pathogenesis of $\mathrm{CM}$ requires further exploration.

Central sensitization is an abnormal state of increased responsiveness of the nociceptive system, which is caused by the enhanced excitability of neurons and circuits in the nociceptive pathway [39]. It is manifested as 

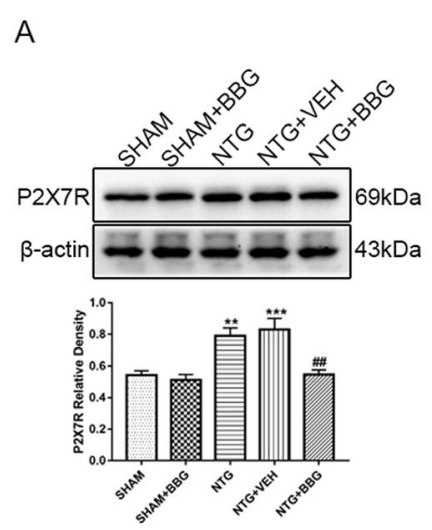

C

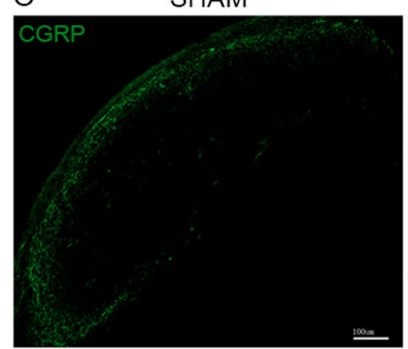

NTG+VEH

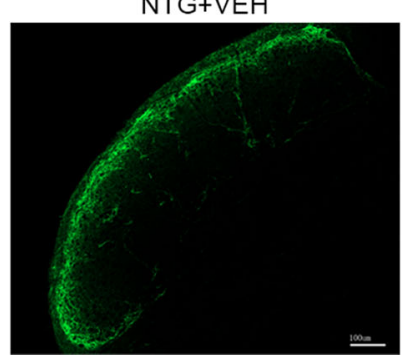

F

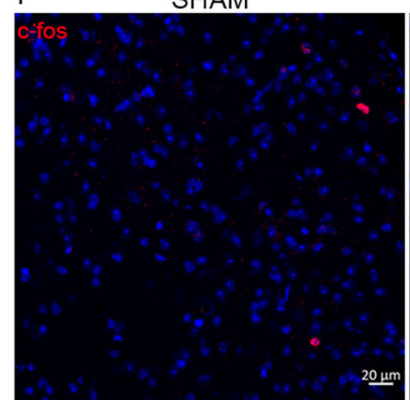

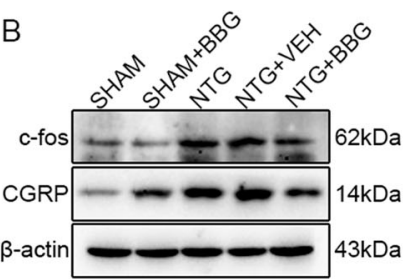

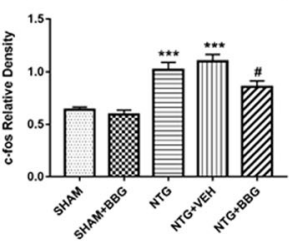

SHAM+BBG

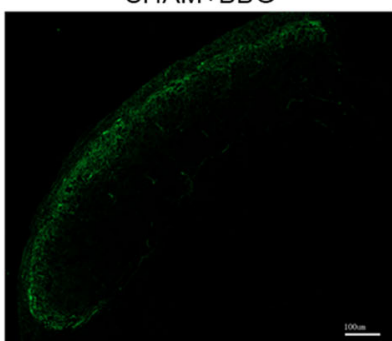

NTG+BBG

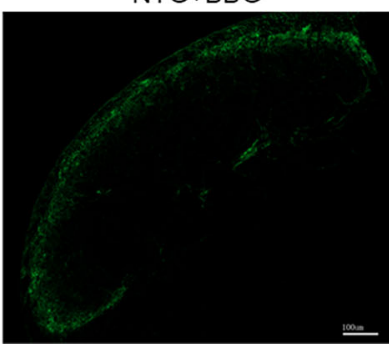

E

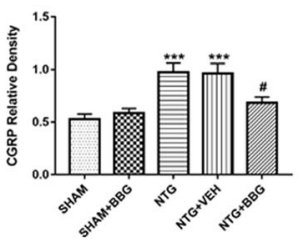

NTG

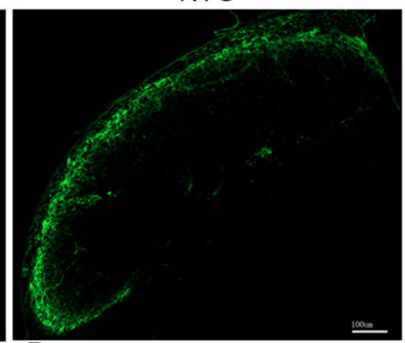

D
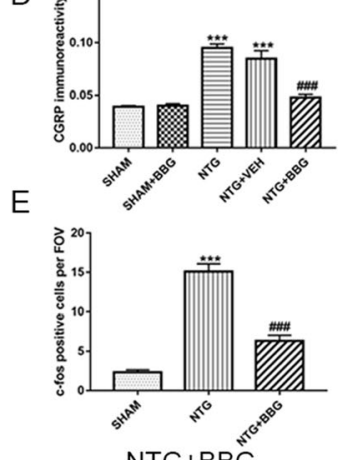

NTG+BBG
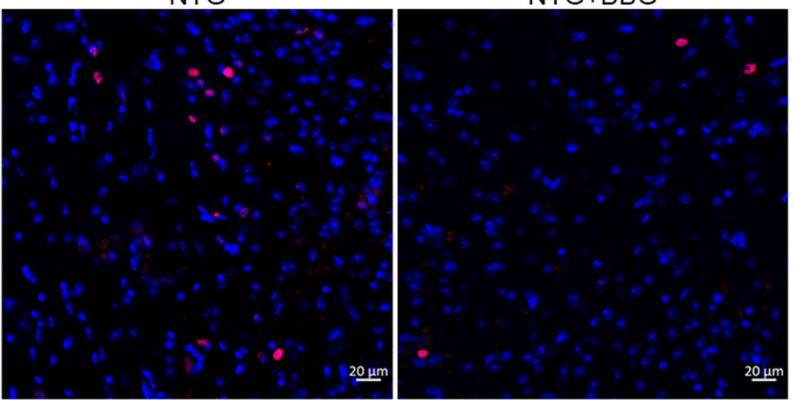

Fig. 8 Inhibiting P2X7R reduced NTG-induced CGRP and c-fos expression in the TNC. a Western blots showing the P2X7R inhibitor, BBG, reduced the expression of P2X7R induced by NTG in the TNC. $\mathbf{b}$ Representative immunoblots of c-fos and CGRP in the TNC after BBG treatment. Quantification of band intensities revealed that BBG partially reversed the upregulation of c-fos and CGRP induced by NTG treatment. $n=6$ per group; Data are presented as mean \pm SEM; one-way ANOVA and Tukey's multiple comparison test; ${ }^{* *} p<0.01$ and ${ }^{* * *} p<0.001$ compared with the SHAM group; ${ }^{\# \#} p<$ 0.01 and ${ }^{\# \# \#} p<0.001$ compared with the NTG group. $\mathbf{c}, \mathbf{f}$ Immunofluorescence staining of CGRP (c) and c-fos (f) in the TNC area. Scale bars: $100 \mu m$ for CGRP, $20 \mu \mathrm{m}$ for c-fos. d, e Quantification of CGRP immunoreactivity (d) and c-fos-positive cells (e) revealed consistent changes with the western blot analysis. $n=4$ per group; 6 sections from each mouse were analyzed; values are expressed as mean \pm SEM; one-way ANOVA and Tukey's multiple comparison test; ${ }^{* * *} p<0.001$ compared with the SHAM group; ${ }^{\# \# \#} p<0.001$ compared with the NTG group 


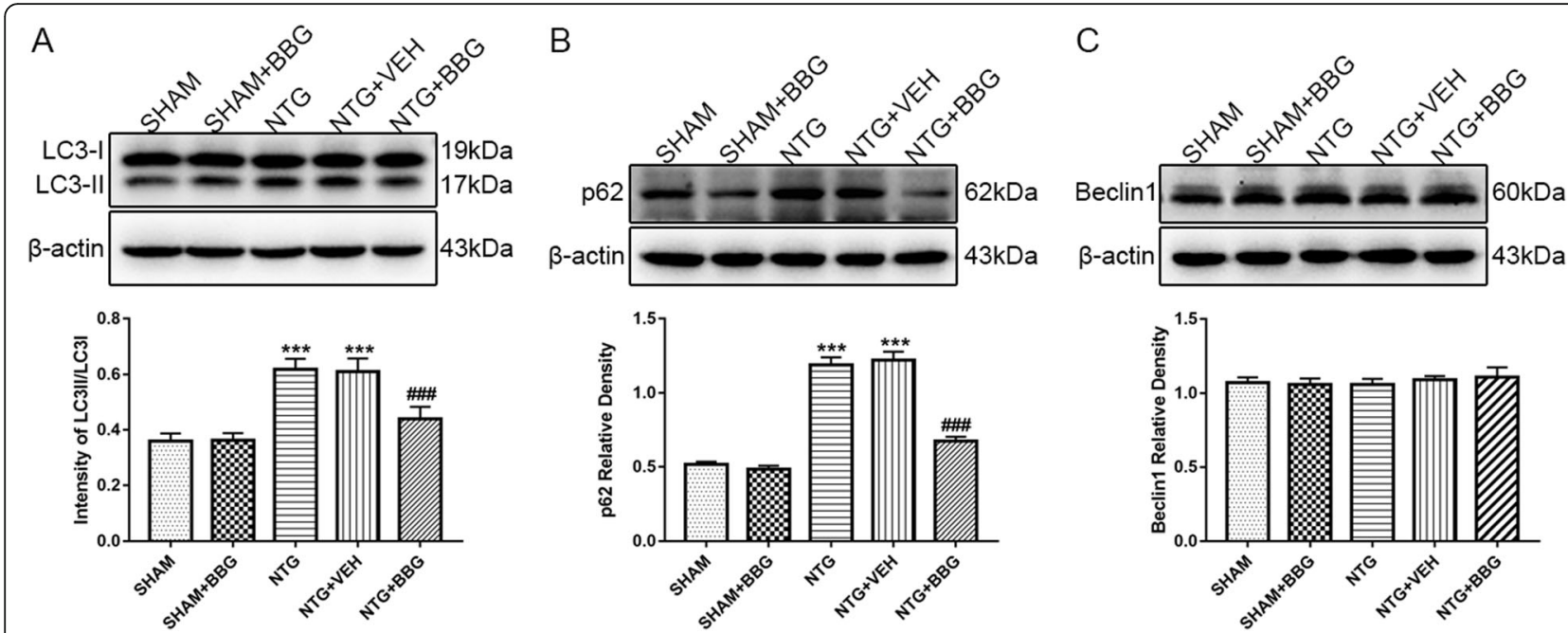

Fig. 9 Blocking P2X7R promoted autophagic flux in the TNC after recurrent NTG injection. a-c Representative western blot of autophagy-related proteins after P2X7R inhibition. $\beta$-actin was used as the control and band density normalization. The optical density analysis showed that after BBG administration, the ratio of LC3II/LC3I (a) decreased, in combination with reduced expression of p62 (b), without any change in beclin1 (c). $n$ $=6$ per group; one-way ANOVA and Tukey's multiple comparison test; data are presented as mean \pm SEM; *** $<0.001$ compared with the SHAM group; $\#$ \# 0.01 compared with the NTG group

spontaneous pain hypersensitivity in response to innocuous stimuli (allodynia) and exaggerated as well as prolonged pain in response to noxious stimuli (hyperalgesia) [39]. Extensive studies have revealed the crucial role of central sensitization as the most important pathophysiology of CM $[3,51]$. CGRP is a very important neuropeptide involved in the development of $\mathrm{CM}$ and has been widely used as the therapeutic target of $\mathrm{CM}$ in the clinic $[37,38]$. It is synthesized by small diameter sensory neurons in the TG, subsequently released and acts on the postsynaptic CGRPR1 on trigeminal neurons in the TNC and thus participates in the central sensitization [38]. c-fos is the protein encoded by the immediate early gene. Its expression level reflects neuronal activity and has been recognized as a reliable marker mediating central sensitization in pain and other nociceptive stimuli $[39,52]$. Accordingly, the expression of CGRP and c-fos were selected to evaluate the central sensitization state, as in our previous studies.

Previous research has explored the pathophysiology of $\mathrm{CM}$, mainly focusing on the neurons in the peripheral nervous system, such as TG. Little attention has been paid to microglia in the CNS. Recently, we found that the activated microglia in the TNC area contribute to central sensitization through releasing the pro-inflammation factors, neurotrophin $[8,10]$. Activation of microglial purinoceptors, P2X4R and P2Y12R, and the NLRP3 inflammasome promoted the development and progression of CM [8-10]. Therefore, the investigation of the underlying molecular mechanism in this study mainly focused on the activation of microglia and the inflammatory response. With regard to the microglia-neuron crosstalk underlying central sensitization, our previous works have provided some explanations.

A large number of studies have suggested that the upregulation of P2X7R is involved in the cancer and neuropathic pain $[13,14]$. Here, we reported increase in the expression of microglial P2X7R in the TNC area and confirmed the relationship between $\mathrm{P} 2 \mathrm{X} 7 \mathrm{R}$ and $\mathrm{CM}$. Chronic administration of the P2X7R antagonist, BBG, significantly alleviated the mechanical and thermal hyperalgesia induced by recurrent NTG injection. In addition, blocking P2X7R also remarkably reduced the expression of CGRP and c-fos, which were selected to evaluate the central sensitization state. We verified the inhibitory effect of P2X7R on the activation of microglia and the NLRP3 inflammasome in the mice model of $\mathrm{CM}$, which was consistent with the results of other neurological diseases reported previously by other teams $[53,54]$. These data indicated that P2X7R blockage may prevent the development of $\mathrm{CM}$ and provided a new target for the CM prophylaxis.

With regard to the cellular localization of P2X7R, P2X7R is initially found to be expressed in peripheral hematopoietic cells, lymphocytes, and macrophages [55]. Following in-depth research, it is believed that $67 \%$ of the P2X7R is also concentrated in the CNS and mainly expressed in glial cells in the CNS, especially microglia $[56,57]$. The presence of P2X7R in neurons has been controversial in the past. However, recent studies have confirmed the expression of P2X7R in neurons, and its activation affects neuronal activity [58]. We observed that P2X7R was mainly expressed in microglia rather than neurons in the TNC. The reasons for this discrepancy 


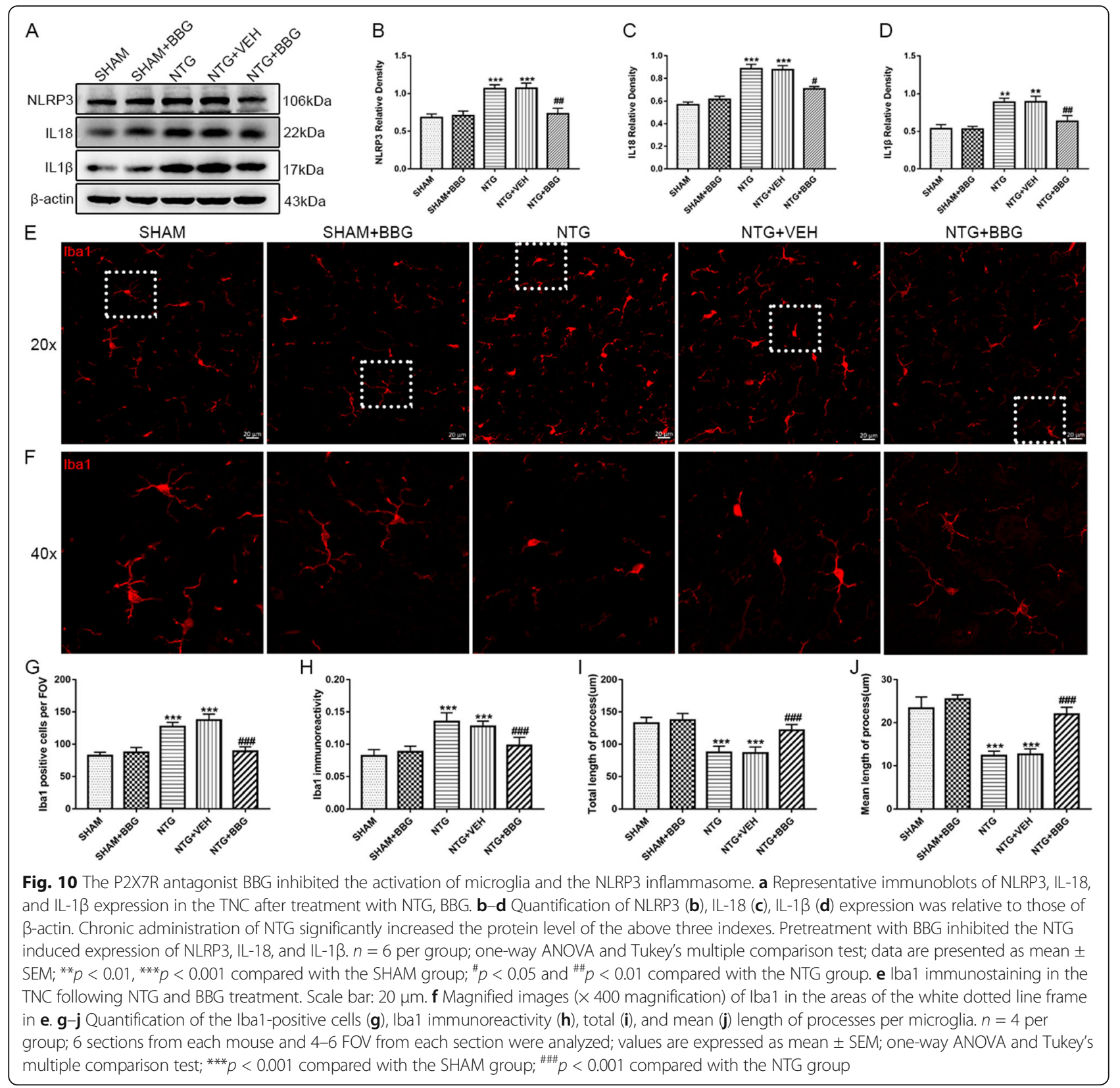

may be due to the different experimental animal model and the different experimental tissue. BBG is a specific P2X7R antagonist that can penetrate the blood-brain barrier [16]. It is well known that BBG at micromolar concentrations also inhibits sodium channels in vitro [59]. However, the dose of BBG used in our experiment was considered to be unable to reach an effective concentration to affect the activity of sodium channels in the brain according to a previous study [60]. Additionally, P2X7R gene knockout was reported to completely abolish the analgesic effect of BBG [16]. Therefore, the BBG used in our experiment was specific to P2X7R. Since BBG was administered systematically in our current study, we cannot rule out the potential effect of other pain modulatory regions exemplified by trigeminal ganglion [61], the diencephalic [62], in CM, because P2X7R is also widely expressed in these areas.

Autophagy is a lysosome-dependent degradation process that breaks down intracellular organelles and misfolded protein aggregates to maintain energy homeostasis and control the quality of the proteins and organelles. Abnormal autophagic processes have been implicated in various degenerative diseases, comprising $\mathrm{AD}, \mathrm{PD}, \mathrm{ALS}$, etc. [63]. Recent evidence has revealed the involvement of autophagy in Nep $[27,28]$. It has been reported that the expression of the autophagy-related protein is altered depending on the different models of Nep [64]. In addition, RAPA, 
a potent autophagy inducer, has been demonstrated to produce a long-lasting analgesic effect on neuropathic pain by inhibiting the activation of microglia, limiting the release of pro-inflammatory factors, and improving nerve myelination [30, 65]. Until now, no research has been conducted to explore the role and specific mechanism of autophagy in migraine. Our current experiment fills this gap.

The LC3-II protein is the most commonly used autophagy marker, and its expression level parallels to the number of autophagosomes [35]. P62 is the endogenous autophagy substrate that recruits the protein for autophagic degradation $[35,36]$. The combination of LC3-II and p62 can preliminarily assess the level of autophagic flux [35]. Our data showed that LC3-II was elevated, accompanied by the accumulation of p62 in the NTGinduced CM model, which indicated the blocked autophagic flux featured by suppression of the degradation route. To further confirm the impaired autophagic flux in $\mathrm{CM}$, we used CQ to inhibit the autophagy-lysosome pathway and compared the expression of LC3-II in NTG-treated mice with and without CQ. The results showed that compared with the mice treated with NTG alone, the protein level of LC3-II did not further increase in the mice treated with NTG and CQ, which also supported the impaired autophagic flux in CM. To further determine the role of autophagy in migraine, we administrated the autophagy inducer, RAPA, and the results showed that RAPA attenuated basal rather than acute hyperalgesia and reduced the expression of CGRP and cfos. These findings supported the preventive function of autophagy activation in CM. In our study, autophagy intervention was achieved through systematic administration of CQ and RAPA, which may also activate potential non-specific effects. To precisely identify the function of autophagy in CM, transgenic mice with autophagic defects are warranted in the future research. In addition, the molecular mechanism underlying autophagy impairment in $\mathrm{CM}$ needs to be further explored.

It is well documented that P2X7R plays a dual role in autophagy pathway regulation depending on the different cell types and stimulation window of P2X7R [22]. In non-transgenic microglia and human epithelial cells, P2X7R negatively regulates autophagy by disrupting lysosomal function $[20,66]$. Nevertheless, in transgenic microglia, exemplified by SOD1-G93A microglia, dystrophic muscle cells, and monocytes as well as macrophages infected with mycobacteria, P2X7R acts as a positive modulator in autophagy [22]. In addition, shortterm stimulation by P2X7R significantly enhances the autophagic flux, while the persistent activation of P2X7R leads to a blockage of autophagic flux [22]. Our present data showed that P2X7R negatively regulated the autophagic flux in CM, which is supported by the reduced expression of LC3-II as well as p62 in response to P2X7R blockage. These results are consistent with those reported by previous studies, since $\mathrm{CM}$ is a wellknown chronic pain and P2X7R is sustainably activated during the chronification process of pain. We did not explore the exact mechanism of P2X7R regulation of autophagy herein. Some evidence has indicated that p38 MAPK can inhibit autophagy by phosphorylating ULK1 $[67,68]$. Meanwhile, the p38 MAPK pathway is the exact downstream of P2X7R in promoting the microglial inflammatory response $[69,70]$. Therefore, we speculated that P2X7R might regulate the autophagic process via the p38 MAPK-ULK1 pathway. Certainly, the specific mechanism requires further exploration.

Substantial investigations have reported the complex reciprocal relationship between autophagy and the microglial inflammatory response [71, 72]. However, no studies have examined this mechanism in migraine. It is well documented that autophagic flux can affect the microglial phenotypes and guide the inflammation into a protective or detrimental state [73, 74]. The inhibited autophagic flux has been reported to induce activation of M1 phenotype microglia and promote the release of pro-inflammatory cytokines. While the enhanced autophagy leads to completely reverse results [40, 41]. Autophagy has been shown to decrease the expression of the NLRP3 inflammasome through regulating the mitochondrial damage and degrading the NLRP3 inflammasome [26]. Our present findings are consistent with the involvement of autophagy in the inflammatory response. We demonstrated that abnormal autophagy might contribute to $\mathrm{CM}$ pathogenesis by regulating the activation of microglia and subsequent inflammatory response.

\section{Conclusions}

In summary, we found a dysfunctional autophagic flux in the TNC and demonstrated the beneficial effect of autophagy induction against $\mathrm{CM}$ progression through inhibiting activation of microglia and production of inflammatory factors. In addition, microglial P2X7R in the TNC was confirmed to participate in the pathogenesis of CM. Blockage of P2X7R alleviated the central sensitization by suppressing microglia and inflammation activation via negatively modulating the autophagic pathway. Our data reveal the modulation of autophagy as a novel mechanism by which P2X7R regulates pain processing. The involvement role of P2X7R and autophagy in $\mathrm{CM}$ introduces new information for investigation of the mechanism of $\mathrm{CM}$ and provides potential therapeutic targets for $\mathrm{CM}$ prevention.

\footnotetext{
Abbreviations

ANOVA: One-way analysis of variance; AD: Alzheimer's disease; BBG: Brilliant Blue G; CM: Chronic migraine; CGRP: Calcitonin gene-related peptide; CNS: Central nervous system; CQ: Chloroquine; FOV: Field of view; Nep: Neuropathic pain; NTG: Nitroglycerin; OD: Optic density; P2X7R: P2X7 receptor; PVDF: Polyvinylidene difluoride; PFA: Paraformaldehyde; PBS: Phosphate-buffered saline; RAPA: Rapamycin; SDS-PAGE: Sodium
} 
dodecyl sulfate-polyacrylamide; SEM: Standard error of the mean; TNC: Trigeminal nucleus caudalis; TBST buffer: Tris-buffered saline with Tween-20

\section{Acknowledgements}

We are grateful to the other staff of the Laboratory Research Center in the First Affiliated Hospital of Chongqing Medical University.

\section{Authors' contributions}

$L J$ designed the study, conducted the experiments, analyzed the data, and drafted the manuscript. FJ, TL, GQ, and DZ provided useful advice on the design of this study, guided the experimental techniques, and helped perform the experiments. JZ and LC handled the funding, supervised the experimental work, and revised this manuscript. All authors read and approved the final manuscript.

\section{Funding}

This study was supported by the National Natural Science Foundation of China [Nos. 81671092 and 81971063].

\section{Availability of data and materials}

The data used in this article are available from the corresponding author on reasonable request if necessary.

\section{Ethics approval and consent to participate}

All animal experiments performed in this study were approved by the Ethics Committee for Animal Experimentation of Chongqing Medical University. The ethical approval reference number is 2020-681.

\section{Consent for publication}

Not applicable.

\section{Competing interests}

The authors declare that they have no competing interests.

\section{Author details}

'Department of Neurology, The First Affiliated Hospital of Chongqing Medical University, 1st You Yi Road, Yuzhong District, Chongqing 400016, China. ${ }^{2}$ Department of Neurology, Chongqing General Hospital, Chongqing, China. ${ }^{3}$ Department of Neurology, The Affiliated Hospital of Southwest Medical University, Luzhou, China. ${ }^{4}$ Laboratory Research Center, The First Affiliated Hospital of Chongqing Medical University, Chongqing, China.

Received: 22 October 2020 Accepted: 9 December 2020

Published online: 05 January 2021

\section{References}

1. (IHS) HCCotlHS. Headache Classification Committee of the International Headache Society (IHS) The International Classification of Headache Disorders, 3rd edition. Cephalalgia. 2018;38:1-211.

2. Agostoni EC, Barbanti $P$, Calabresi $P$, Colombo B, Cortelli P, Frediani F, Geppetti P, Grazzi L, Leone M, Martelletti $P$, et al. Current and emerging evidence-based treatment options in chronic migraine: a narrative review. J Headache Pain. 2019;20:92.

3. Su M, Yu S. Chronic migraine: a process of dysmodulation and sensitization. Mol Pain. 2018;14:1744806918767697.

4. Boyer N, Dallel R, Artola A, Monconduit L. General trigeminospinal central sensitization and impaired descending pain inhibitory controls contribute to migraine progression. Pain. 2014;155:1196-205.

5. Edvinsson L, Haanes KA, Warfvinge K. Does inflammation have a role in migraine? Nat Rev Neurol. 2019;15:483-90.

6. Magni G, Pedretti S, Audano M, Caruso D, Mitro N, Ceruti S. Glial cell activation and altered metabolic profile in the spinal-trigeminal axis in a rat model of multiple sclerosis associated with the development of trigeminal sensitization. Brain Behav Immun. 2020;89:268-80.

7. Long T, He W, Pan Q, Zhang S, Zhang Y, Liu C, Liu Q, Qin G, Chen L, Zhou J. Microglia P2X4 receptor contributes to central sensitization following recurrent nitroglycerin stimulation. J Neuroinflammation. 2018;15:245.

8. Long T, He W, Pan Q, Zhang S, Zhang D, Qin G, Chen L, Zhou J. Microglia P2X4R-BDNF signalling contributes to central sensitization in a recurrent nitroglycerin-induced chronic migraine model. J Headache Pain. 2020;21:4.
9. Jing F, Zhang Y, Long T, He W, Qin G, Zhang D, Chen L, Zhou J. P2Y12 receptor mediates microglial activation via RhoA/ROCK pathway in the trigeminal nucleus caudalis in a mouse model of chronic migraine. J Neuroinflammation. 2019;16:217.

10. He W, Long T, Pan Q, Zhang S, Zhang Y, Zhang D, Qin G, Chen L, Zhou J. Microglial NLRP3 inflammasome activation mediates IL-1beta release and contributes to central sensitization in a recurrent nitroglycerin-induced migraine model. J Neuroinflammation. 2019;16:78.

11. Burnstock G. Pathophysiology of migraine: a new hypothesis. Lancet. 1981;1: 1397-9.

12. Bhattacharya A, Biber K. The microglial ATP-gated ion channel P2X7 as a CNS drug target. Glia. 2016;64:1772-87.

13. Yang Y, Li H, Li TT, Luo H, Gu XY, Lu N, Ji RR, Zhang YQ. Delayed activation of spinal microglia contributes to the maintenance of bone cancer pain in female Wistar rats via P2X7 receptor and IL-18. J Neurosci. 2015;35:7950-63.

14. Hu X, Liu Y, Wu J, Liu Y, Liu W, Chen J, Yang F. Inhibition of P2X7R in the amygdala ameliorates symptoms of neuropathic pain after spared nerve injury in rats. Brain Behav Immun. 2020;88:507-14.

15. Liu PY, Lee $\mid H$, Tan PH, Wang YP, Tsai CF, Lin HC, Lee FY, Lu CL. P2X7 Receptor Mediates Spinal Microglia Activation of Visceral Hyperalgesia in a Rat Model of Chronic Pancreatitis. Cell Mol Gastroenterol Hepatol. 2015;1: 710-20 e715.

16. Goloncser F, Sperlagh B. Effect of genetic deletion and pharmacological antagonism of P2X7 receptors in a mouse animal model of migraine. J Headache Pain. 2014;15:24

17. Mizushima N. A brief history of autophagy from cell biology to physiology and disease. Nat Cell Biol. 2018;20:521-7.

18. Clarke AJ, Simon AK. Autophagy in the renewal, differentiation and homeostasis of immune cells. Nat Rev Immunol. 2019;19:170-83.

19. Deretic V, Saitoh T, Akira S. Autophagy in infection, inflammation and immunity. Nat Rev Immunol. 2013;13:722-37.

20. Takenouchi T, Fujita M, Sugama S, Kitani H, Hashimoto M. The role of the P2X7 receptor signaling pathway for the release of autolysosomes in microglial cells. Autophagy. 2009;5:723-4.

21. Kim JE, Ko AR, Hyun HW, Min SJ, Kang TC. P2RX7-MAPK1/2-SP1 axis inhibits MTOR independent HSPB1-mediated astroglial autophagy. Cell Death Dis. 2018;9:546.

22. Fabbrizio P, Amadio S, Apolloni S, Volonte C. P2X7 Receptor Activation Modulates Autophagy in SOD1-G93A Mouse Microglia. Front Cell Neurosci. 2017;11:249.

23. Young CN, Sinadinos A, Lefebvre A, Chan P, Arkle S, Vaudry D, Gorecki DC. A novel mechanism of autophagic cell death in dystrophic muscle regulated by P2RX7 receptor large-pore formation and HSP90. Autophagy. 2015:11:113-30.

24. Houtman J, Freitag K, Gimber N, Schmoranzer J, Heppner FL, Jendrach M. Beclin1-driven autophagy modulates the inflammatory response of microglia via NLRP3. EMBO J. 2019;38.

25. Su P, Zhang J, Wang D, Zhao F, Cao Z, Aschner M, Luo W. The role of autophagy in modulation of neuroinflammation in microglia. Neuroscience. 2016:319:155-67.

26. Cho MH, Cho K, Kang HJ, Jeon EY, Kim HS, Kwon HJ, Kim HM, Kim DH, Yoon SY. Autophagy in microglia degrades extracellular beta-amyloid fibrils and regulates the NLRP3 inflammasome. Autophagy. 2014;10:1761-75.

27. Liu X, Zhu M, Ju Y, Li A, Sun X. Autophagy dysfunction in neuropathic pain. Neuropeptides. 2019;75:41-8.

28. Weng W, Yao C, Poonit K, Zhou X, Sun C, Zhang F, Yan H. Metformin relieves neuropathic pain after spinal nerve ligation via autophagy flux stimulation. J Cell Mol Med. 2019;23:1313-24.

29. Pradhan AA, Smith ML, McGuire B, Tarash I, Evans CJ, Charles A. Characterization of a novel model of chronic migraine. Pain. 2014;155: 269-74.

30. Tateda S, Kanno H, Ozawa H, Sekiguchi A, Yahata K, Yamaya S, Itoi E. Rapamycin suppresses microglial activation and reduces the development of neuropathic pain after spinal cord injury. J Orthop Res. 2017;35:93-103.

31. Zheng Z, Wu Y, Li Z, Ye L, Lu Q, Zhou Y, Yuan Y, Jiang T, Xie L, Liu Y, et al. Valproic acid affects neuronal fate and microglial function via enhancing autophagic flux in mice after traumatic brain injury. J Neurochem. 2020;154: 284-300.

32. Louter MA, Bosker JE, van Oosterhout WP, van Zwet EW, Zitman FG, Ferrari MD, Terwindt GM. Cutaneous allodynia as a predictor of migraine chronification. Brain. 2013;136:3489-96. 
33. Bates EA, Nikai T, Brennan KC, Fu YH, Charles AC, Basbaum Al, Ptacek L, Ahn AH. Sumatriptan alleviates nitroglycerin-induced mechanical and thermal allodynia in mice. Cephalalgia. 2010;30:170-8.

34. Pinskiy V, Tolpygo AS, Jones J, Weber K, Franciotti N, Mitra PP. A low-cost technique to cryo-protect and freeze rodent brains, precisely aligned to stereotaxic coordinates for whole-brain cryosectioning. J Neurosci Methods. 2013;218:206-13.

35. Klionsky DJ, Abdalla FC, Abeliovich H, Abraham RT, Acevedo-Arozena A, Adeli K, Agholme L, Agnello M, Agostinis P, Aguirre-Ghiso JA, et al. Guidelines for the use and interpretation of assays for monitoring autophagy. Autophagy. 2012:8:445-544.

36. Ichimura $Y$, Komatsu M. Selective degradation of $\mathrm{p} 62$ by autophagy. Semin Immunopathol. 2010;32:431-6.

37. lyengar S, Johnson KW, Ossipov MH, Aurora SK. CGRP and the Trigeminal System in Migraine. Headache. 2019;59:659-81.

38. lyengar S, Ossipov MH, Johnson KW. The role of calcitonin gene-related peptide in peripheral and central pain mechanisms including migraine. Pain. 2017;158:543-59.

39. Latremoliere A, Woolf CJ. Central sensitization: a generator of pain hypersensitivity by central neural plasticity. J Pain. 2009;10:895-926.

40. Plaza-Zabala A, Sierra-Torre V, Sierra A. Autophagy and Microglia: Novel Partners in Neurodegeneration and Aging. Int J Mol Sci. 2017;18.

41. Ji J, Xue TF, Guo XD, Yang J, Guo RB, Wang J, Huang JY, Zhao XJ, Sun XL. Antagonizing peroxisome proliferator-activated receptor gamma facilitates M1-to-M2 shift of microglia by enhancing autophagy via the LKB1-AMPK signaling pathway. Aging Cell. 2018;17:e12774.

42. Akerman S, Karsan N, Bose P, Hoffmann JR, Holland PR, Romero-Reyes M, Goadsby PJ. Nitroglycerine triggers triptan-responsive cranial allodynia and trigeminal neuronal hypersensitivity. Brain. 2019;142:103-19.

43. Schoonman GG, Sandor PS, Agosti RM, Siccoli M, Bartsch P, Ferrari MD, Baumgartner RW. Normobaric hypoxia and nitroglycerin as trigger factors for migraine. Cephalalgia. 2006;26:816-9.

44. Uglem M, Omland PM, Nilsen KB, Tronvik E, Stovner LJ, Hagen K, Linde M, Sand T. Does pain sensitivity change by migraine phase? A blinded longitudinal study. Cephalalgia. 2017;37:1337-49.

45. Markovics A, Kormos V, Gaszner B, Lashgarara A, Szoke E, Sandor K, Szabadfi K, Tuka B, Tajti J, Szolcsanyi J, et al. Pituitary adenylate cyclase-activating polypeptide plays a key role in nitroglycerol-induced trigeminovascular activation in mice. Neurobiol Dis. 2012;45:633-44.

46. Tang Y, Liu S, Shu H, Xing Y, Tao F. AMPA receptor GluA1 Ser831 phosphorylation is critical for nitroglycerin-induced migraine-like pain. Neuropharmacology. 2018;133:462-9.

47. Brandes $J \mathrm{~L}$. The influence of estrogen on migraine: a systematic review. JAMA. 2006;295:1824-30

48. Chai NC, Peterlin BL, Calhoun AH. Migraine and estrogen. Curr Opin Neurol. 2014;27:315-24.

49. Craft RM. Modulation of pain by estrogens. Pain. 2007;132(Suppl 1):S3-12.

50. Amandusson A, Blomqvist A. Estrogenic influences in pain processing. Front Neuroendocrinol. 2013;34:329-49.

51. Dodick D, Silberstein S. Central sensitization theory of migraine: clinical implications. Headache. 2006;46(Suppl 4):S182-91.

52. Hermanson O, Telkov M, Geijer T, Hallbeck M, Blomqvist A. Preprodynorphin mRNA-expressing neurones in the rat parabrachial nucleus: subnuclear localization, hypothalamic projections and colocalization with noxiousevoked fos-like immunoreactivity. Eur J Neurosci. 1998;10:358-67.

53. Yue N, Huang H, Zhu X, Han Q, Wang Y, Li B, Liu Q, Wu G, Zhang Y, Yu J. Activation of P2X7 receptor and NLRP3 inflammasome assembly in hippocampal glial cells mediates chronic stress-induced depressive-like behaviors. J Neuroinflammation. 2017;14:102.

54. Zhao H, Pan P, Yang Y, Ge H, Chen W, Qu J, Shi J, Cui G, Liu X, Feng H, Chen $Y$. Endogenous hydrogen sulphide attenuates NLRP3 inflammasomemediated neuroinflammation by suppressing the $\mathrm{P} 2 \mathrm{X} 7$ receptor after intracerebral haemorrhage in rats. J Neuroinflammation. 2017;14:163.

55. Filippin KJ, de Souza KFS, de Araujo Junior RT, Torquato HFV, Dias DA, Parisotto EB, Ferreira AT, Paredes-Gamero EJ. Involvement of P2 receptors in hematopoiesis and hematopoietic disorders, and as pharmacological targets. Purinergic Signal. 2020;16:1-15.

56. He Y, Taylor N, Fourgeaud L, Bhattacharya A. The role of microglial P2X7: modulation of cell death and cytokine release. J Neuroinflammation. 2017;14:135.

57. Kaczmarek-Hajek K, Zhang J, Kopp R, Grosche A, Rissiek B, Saul A, Bruzzone S, Engel T, Jooss T, Krautloher A, et al. Re-evaluation of neuronal P2X7 expression using novel mouse models and a P2X7specific nanobody. Elife. 2018;7.

58. Illes P, Khan TM, Rubini P. Neuronal P2X7 Receptors Revisited: Do They Really Exist? J Neurosci. 2017;37:7049-62.

59. Jo S, Bean BP. Inhibition of neuronal voltage-gated sodium channels by brilliant blue G. Mol Pharmacol. 2011:80:247-57.

60. Diaz-Hernandez Jl, Gomez-Villafuertes R, Leon-Otegui M, Hontecillas-Prieto L, Del Puerto A, Trejo JL, Lucas JJ, Garrido JJ, Gualix J, Miras-Portugal MT, DiazHernandez M. In vivo P2X7 inhibition reduces amyloid plaques in Alzheimer's disease through GSK3beta and secretases. Neurobiol Aging. 2012:33:1816-28.

61. Nowodworska A, van den Maagdenberg A, Nistri A, Fabbretti E. In situ imaging reveals properties of purinergic signalling in trigeminal sensory ganglia in vitro. Purinergic Signal. 2017;13:511-20.

62. Akerman S, Holland PR, Goadsby PJ. Diencephalic and brainstem mechanisms in migraine. Nat Rev Neurosci. 2011;12:570-84.

63. Moloudizargari M, Asghari MH, Ghobadi E, Fallah M, Rasouli S, Abdollahi M. Autophagy, its mechanisms and regulation: Implications in neurodegenerative diseases. Ageing Res Rev. 2017:40:64-74.

64. Berliocchi L, Maiaru M, Varano GP, Russo R, Corasaniti MT, Bagetta G, Tassorelli C. Spinal autophagy is differently modulated in distinct mouse models of neuropathic pain. Mol Pain. 2015;11:3.

65. Rangaraju S, Verrier JD, Madorsky I, Nicks J, Dunn WA Jr, Notterpek L. Rapamycin activates autophagy and improves myelination in explant cultures from neuropathic mice. J Neurosci. 2010;30:11388-97.

66. Haanes KA, Schwab A, Novak I. The P2X7 receptor supports both life and death in fibrogenic pancreatic stellate cells. PLoS One. 2012;7:e51164.

67. He Y, She H, Zhang T, Xu H, Cheng L, Yepes M, Zhao Y, Mao Z. p38 MAPK inhibits autophagy and promotes microglial inflammatory responses by phosphorylating ULK1. J Cell Biol. 2018;217:315-28.

68. She H, He Y, Zhao Y, Mao Z. Release the autophage brake on inflammation: the MAPK14/p38alpha-ULK1 pedal. Autophagy. 2018;14:1097-8.

69. Zhang QL, Wang W, Jiang Y, Tuya A, Dongmei LLL, Lu ZJ, Chang H, Zhang TZ. GRGM-13 comprising 13 plant and animal products, inhibited oxidative stress induced apoptosis in retinal ganglion cells by inhibiting P2RX7/p38 MAPK signaling pathway. Biomed Pharmacother. 2018;101:494-500.

70. Chen S, Ma Q, Krafft PR, Chen Y, Tang J, Zhang J, Zhang JH. P2X7 receptor antagonism inhibits p38 mitogen-activated protein kinase activation and ameliorates neuronal apoptosis after subarachnoid hemorrhage in rats. Crit Care Med. 2013;41:e466-74.

71. Deretic V, Klionsky DJ. Autophagy and inflammation: a special review issue. Autophagy. 2018;14:179-80.

72. Matsuzawa-Ishimoto Y, Hwang S, Cadwell K. Autophagy and inflammation. Annu Rev Immunol. 2018;36:73-101.

73. Jin MM, Wang F, Qi D, Liu WW, Gu C, Mao CJ, Yang YP, Zhao Z, Hu LF, Liu CF. A critical role of autophagy in regulating microglia polarization in neurodegeneration. Front Aging Neurosci. 2018;10:378.

74. Hu ZW, Zhou LQ, Yang S, Chen M, Yu HH, Tao R, Wu L, Wang W, Zhang Q, Qin C, Tian DS. FTY720 Modulates microglia toward anti-inflammatory phenotype by suppressing autophagy via STAT1 pathway. Cell Mol Neurobiol. 2020; https://doi.org/10.1007/s10571-020-00856-9.

\section{Publisher's Note}

Springer Nature remains neutral with regard to jurisdictional claims in published maps and institutional affiliations.

\section{Ready to submit your research? Choose BMC and benefit from:}

- fast, convenient online submission

- thorough peer review by experienced researchers in your field

- rapid publication on acceptance

- support for research data, including large and complex data types

- gold Open Access which fosters wider collaboration and increased citations

- maximum visibility for your research: over $100 \mathrm{M}$ website views per year

At BMC, research is always in progress.

Learn more biomedcentral.com/submission 